\title{
Origin and Quantification of Increased Core Loss in MnZn Ferrite Plates of a Multi-Gap Inductor
}

\author{
Dominik Neumayr, Dominik Bortis, Johann W. Kolar, Stefan Hoffmann, and Eckart Hoene
}

\begin{abstract}
Inductors realized with high permeable $\mathrm{MnZn}$ ferrite require, unlike iron-powder cores with an inherent distributed gap, a discrete air gap in the magnetic circuit to prevent saturation of the core material and/or tune the inductance value. This large discrete gap can be divided into several partial gaps in order to reduce the air gap stray field and consequently the proximity losses in the winding. The multi-gap core, realized by stacking several thin ferrite plates and inserting a non-magnetic spacer material between the plates, however, exhibits a substantial increase in core losses which cannot be explained from the intrinsic properties of the ferrite. In this paper, a comprehensive overview of the scientific literature regarding machining induced core losses in ferrite, dating back to the early 1970s, is provided which suggests that the observed excess core losses could be attributed to a deterioration of ferrite properties in the surface layer of the plates caused by mechanical stress exerted during machining. However, in a first experimental analysis no structural evidence for a deteriorated layer close to the surface is identified by means of Scanning Electron Microscopy (SEM). Therefore, in a next step, a new calorimetric measurement setup based on temperature rise monitoring is proposed in this paper in order to quantify and differentiate between core losses associated with the bulk and the surface of the ferrite plates and therefore to pinpoint the measured excess core loss to shallow layers of ferrite with deteriorated magnetic performance. Electrical measurement of the surface related core losses utilizing the widely accepted twowinding wattmeter method with reactive power compensation is outlined in the appendix but was not employed in this work due to comparably low measurement accuracy. By means of the proposed measurement technique, the bulk and surface core loss density of the MnZn ferrite material 3F4 from FerroxCube was determined for sinusoidal flux density amplitude varying from $75 \mathrm{mT}$ up to $200 \mathrm{mT}$ and excitation frequencies ranging from $200 \mathrm{kHz}$ to $1 \mathrm{MHz}$. The measured core loss densities $\left(\mathrm{W} / \mathrm{cm}^{3}\right)$ show good agreement with the Steinmetz model provided by the manufacturer validating the proposed calorimetric core loss measurement technique. The measured surface loss density $\left(\mathrm{W} / \mathrm{cm}^{2}\right)$ can also be well predicted with a Steinmetz model, whereby the frequency exponent $\alpha$ in the surface is slightly smaller and the flux density exponent $\beta$ is slightly larger compared to the Steinmetz parameter of the bulk ferrite. It is shown that the ratio between surface and bulk core losses of a composite core assembled from individual plates is only a function of plate thickness and does not depend on the actual cross section
\end{abstract}

Manuscript received July 6, 2018. (Corresponding author: Dominik Neumayr.)

D. Neumayr, D. Bortis, and J. W. Kolar are with the Power Electronic Systems Laboratory, ETH Zurich, ETL I12, Physikstrasse 3, 8092 Zurich, Switzerland (e-mail: neumayr@lem.ee.ethz.ch).

S. Hoffmann and E. Hoene are with Fraunhofer Institute for Reliability and Microintegration (IZM), Berlin, Germany.

Digital Object Identifier 10.24295/CPSSTPEA.2019.00008 area. Critical plate thickness is then defined to be reached when the total power loss in the composite core has doubled compared to a solid (single-piece) core sample. This new quantity provides a very helpful figure for multi-gap inductor designs. Besides the deteriorated surface layers, several other mechanisms potentially contributing to increased core losses in multi-gap inductors were identified and are finally discussed in the appendix of this paper: flux crowding in the core due to tolerances and imperfections in machining and assembly; deterioration of ferrite properties due to pressure buildup in the stack of plates during the curing of the employed epoxy resin; ohmic loss in the ferrite associated with the current flowing in the conduction path provided by the low impedance of the ferrite material at high frequencies and the parasitic capacitance between winding and the ferrite core.

Index Terms - Distributed air gap, high $\mathrm{Q}$ inductor, machining induced loss, MnZn ferrite, multi-gap inductor, surface loss.

\section{INTRODUCTION}

$I$ high power density converter systems, the switching frequency is increased substantially in order to strongly reduce the required inductance value and the volume of the employed power inductor. Very compact input inductors of PFC rectifiers and output inductors of inverters or DC/DC converters also feature a high current ripple which demands suitable core materials - at high frequencies preferable ferrite - and sophisticated inductor designs in order to keep the core and winding losses to a minimum. Inductors realized with high permeable ferrite materials require, unlike iron-powder cores with an inherent distributed gap, a discrete air gap in the magnetic circuit to prevent saturation of the core material and/ or tune the inductance value. This large discrete air gap can be divided into several partial gaps in order to reduce the air gap stray field and consequently the proximity losses in the winding, especially if copper foil is used to implement the winding (cf., Fig. 1(a) and (b)). If the partial gaps are distributed over the entire length of the inner limb of an E-type core, the $\mathrm{H}$-field in the winding window shows a quasi one-dimensional field distribution running in parallel to the inner limb [1]-[3] This allows to implement the winding with copper foil which achieves a higher filling factor compared to HF litz wire, since the individual layers of the foil winding are aligned in parallel with the H-field and excessive eddy current losses can be avoided.

Following this idea, ETH Zurich together with Fraunhofer 


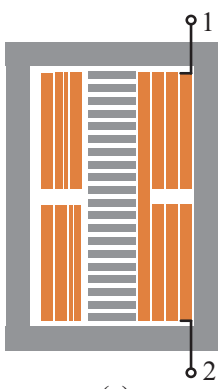

(a)
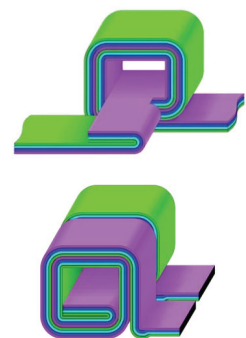

(b)

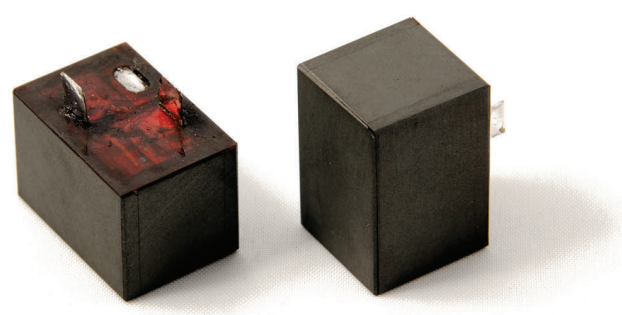

(c)

Fig. 1. (a) Structure of the foil winding multi-gap inductor with the center limb of the E-type core being composed of stacked ferrite plates. (b) Foil winding arrangement to achieve equal current sharing in parallel foils which has both connection terminals at the outside of the winding [4], [5]. (c) Hardware picture of the realized $10.5 \mu \mathrm{H}$ multi-gap HF inductor employed in the Google Little Box Challenge converter [6].

Institute for Reliability and Microintegration (IZM) and Fraza company developed compact multi-gap foil winding inductors to realize a $8.2 \mathrm{~kW} / \mathrm{dm}^{3}\left(134 \mathrm{~W} / \mathrm{in}^{3}\right)$ single-phase, fullbridge based PV inverter for the GOOGLE and IEEE Little Box Challenge (LBC) targeting the realization of the world smallest $2 \mathrm{~kW}$ inverter [7]. In order to achieve soft-switching throughout the mains period, Triangular Current Mode (TCM) modulation was employed resulting in a variable switching frequency in the range of $200 \mathrm{kHz}-1 \mathrm{MHz}$ for an inductance value of $10.5 \mu \mathrm{H}$. These system parameters were obtained from a Pareto optimization as described in detail in [6]. A picture of the realized inductor with dimension $(14.5 \times 14.5 \times 22) \mathrm{mm}^{3}$ is shown in Fig. 1(c). The inner limb of the inductor is composed of $24 \times 0.6 \mathrm{~mm}$ thick stacked ferrite plates with $80 \mu \mathrm{m}$ partial air gaps between the plates, resulting in a total air gap length of $1.92 \mathrm{~mm}$. The inner limb is surrounded by the foil winding with a total of 16 turns, where each turn is composed of four parallel $20 \mu \mathrm{m}$ thin copper foils which are mutually isolated with a $7 \mu \mathrm{m}$ thin layer of Kapton. Furthermore, a sophisticated winding arrangement is used as shown in Fig. 1(b), which forces the current to flow evenly distributed in all four parallel copper layer, thus counteracting the skin and proximity effect [4], [5], [8], [9]. Moreover, the arrangement features both connection terminals at the outside of the winding where the magnitude of the $\mathrm{H}$-field in the winding window is small, reducing losses caused by the termination of the foil winding, where it is difficult to keep the copper foil aligned with the $\mathrm{H}$-field, to a minimum. The used core material is DMR 51 from the manufacturer DMEGC, a MnZn ferrite optimized for the frequency range of $1 \mathrm{MHz}-2 \mathrm{MHz}$ (similar in performance to
TABLE I

Technical Data of the Multi-Gap Foll Winding Inductor

\begin{tabular}{ll}
\hline \hline Inductance & $10.5 \mu \mathrm{H}$ \\
Quality factor & $\approx 600$ between $200 \mathrm{kHz}-1000 \mathrm{kHz}$ \\
$R_{\mathrm{AC}}$ & $14 \mathrm{~m} \Omega-120 \mathrm{~m} \Omega$ between $\mathrm{DC}-1 \mathrm{MHz}$ \\
Dimension & $(14.5 \times 14.5 \times 22) \mathrm{mm}^{3}$ \\
\hline \multirow{3}{*}{ Foil Winding } & $2 \times 8$ turns \\
& $4 \mu \mathrm{p}$ Kallel $20 \mu \mathrm{m}$ copper foils \\
& $\begin{array}{l}\text { MnZn Ferrite DMR } 51 / \text { DMEGC } \\
\text { Core }\end{array}$ \\
& $24 \times 80 \mu \mathrm{m}$ air gaps \\
& $0.61 \mathrm{~mm}$ thick stacked plates \\
& $(6.6 \times 9.6 \times 17.5) \mathrm{mm}^{3}$ center limb dimension \\
\hline \hline
\end{tabular}

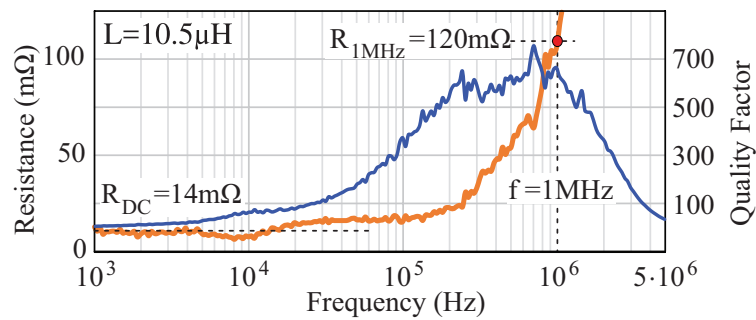

(a)

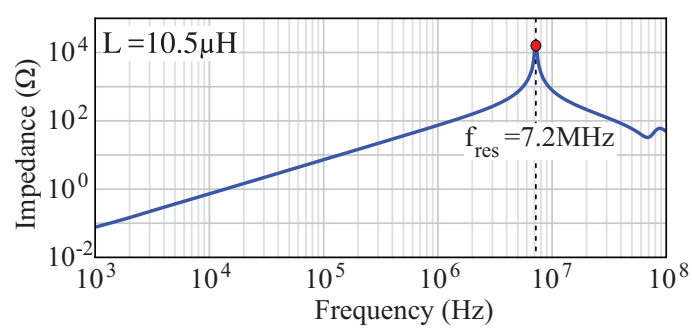

(b)

Fig. 2. Small-signal impedance analyzer measurements with Agilent 4294A of the multi-gap inductor depicted in Fig. 1(c). (a) Winding resistance and quality factor with respect to frequency. (b) Impedance with respect to frequency.

3F4 from Ferroxcube or PC200 from TDK).

All technical specifications of the multi-gap inductor are summarized in Table I and impedance analyzer measurements are presented in Fig. 2. It can be seen that the implemented multi-gap foil winding inductor exhibits an excellent quality factor of $600-700$ between $200 \mathrm{kHz}-1 \mathrm{MHz}$ and a high resonance frequency of around $7.2 \mathrm{MHz}$.

Unfortunately, experimental measurements at an early stage of development revealed a substantially higher power loss than expected. By means of core loss measurements and thermographic inspection, the origin of additional losses could be attributed to the stacked ferrite plates constituting the center limb of the core. For this reason, the number of partial gaps was reduced from initially 50 to just 24 in the final inductor design (cf., Table I). Upon consultation of scientific literature and further experimental investigation, four main reasons for the increased core losses were identified:

- Formation of deteriorated ferrite layers at the plate surfaces due to mechanical stress introduced during machining. 
- Flux crowding in the multi-gap core due to tolerances and imperfections in machining and assembly (Appendix A).

- Deterioration of ferrite properties due to pressure buildup in the stack of plates during the curing of the employed epoxy resin (Appendix B).

- Ohmic loss in the ferrite associated with the current flowing in the conduction path provided by the low impedance of the ferrite material at high frequencies and the parasitic capacitance between outer layer of the foil winding and the ferrite (Appendix C).

For the sake of brevity, only the surface loss related mechanism will be addressed in detail in this paper since it is by far the most significant contributor to the excess core loss. However, the interested reader is referred to the respective Appendix of this paper for a brief discussion of the other loss mechanism. The main scientific contribution of this work is an experimental method which allows to differentiate between core losses associated with the bulk and the surface of the ferrite plates required to implement the multi-gap inductor design and thus to pinpoint the measured excess core loss to shallow surface layers of ferrite with deteriorated magnetic performance as suggested in scientific literature dating back to the ' 70 s. Moreover, the proposed technique allows to assess modern ferrite materials regarding their applicability in multi-gap inductor designs.

In Section II of this paper, the most important analyses and findings are shown in the literature regarding the impact of mechanical processing, such as cutting and grinding on the magnetic properties of ferrite are summarized and a microstructural analysis of the machined ferrite surface is presented. In Section III, mathematical expressions are derived to quantify core and surface loss densities from experimental temperature rise measurements using multi-gap core samples assembled from machined ferrite plates with distinct thicknesses. Moreover, the employed experimental setup based on Infrared (IR) thermometry is outlined. The proposed technique is then applied to characterize the MnZn ferrite material 3F4 at several peak flux densities and frequency values, that is to say at operating points $\{\hat{B}, f\}$ which resemble the Google Little Box Challenge application. The obtained experimental findings are presented in Section IV and subsequently discussed in Section V where the ratio between bulk and surface core loss is illustrated. Section VI concludes the paper and provides an outlook for future research.

\section{Machining Induced InCREASE OF CORE Loss}

The correct dimensions of ferrite plates or cuboidal pieces needed to implement a multi-gap core are typically not off-the-shelf available and must be machined from a larger ferrite piece. One option is to cut the plates from a long sintered ferrite bar using a precision saw as depicted in Fig. 3(a). Since ferrite is a very hard and brittle ceramic, the saw must be equipped with a diamond blade. As a consequence of the cutting process with the abrasive diamond particles, a mechanical stress is introduced into the ground surface of the plate. As will be summarized in the following, it is claimed in the scientific literature that the exerted

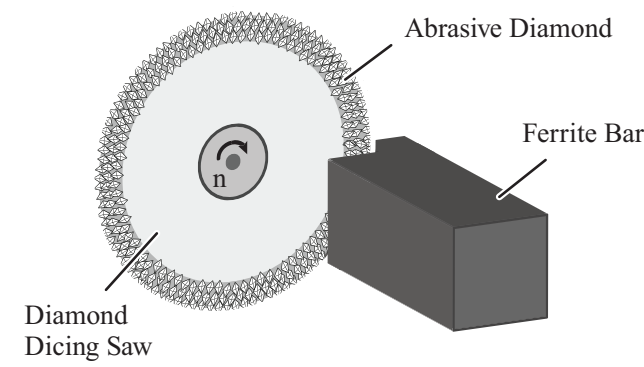

(a)

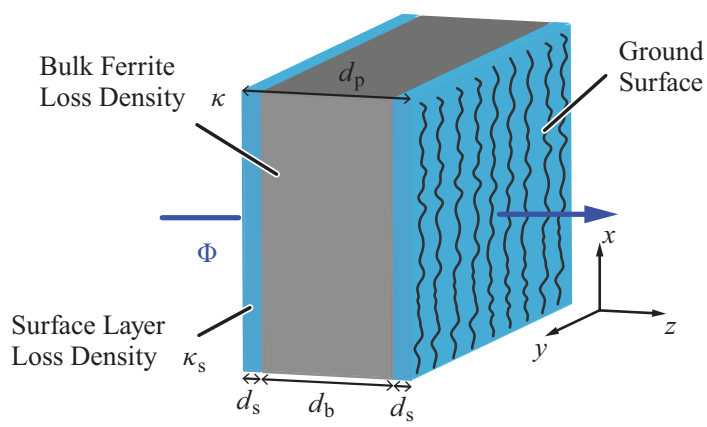

(b)

Fig. 3. (a) Cutting thin ferrite plates or cuboidal pieces from a long bar using a precision saw equipped with a diamond blade. (b) Abrasive machining causes residual mechanical stress in the ground surface; the magnetic properties of ferrite are degraded in these shallow layers and feature a much higher core loss density as opposed to the ferrite with intrinsic properties in the bulk of the plate.

mechanical stress is deteriorating the magnetic properties of the ferrite in a thin layer just underneath the surface resulting in an increase of overall core loss (cf., Fig. 3(b)).

The first investigations regarding the impact of mechanical stress on the properties of ferrite were conducted by E. Stern and D. Temme back in 1964 [10]. They observed that mechanical pressure applied to a toroidal core changes the remanent magnetization, coercive force and the shape of the BH-loop. They also claimed that compressive stress can result from abrasive machining and predominantly affects the ferrite close to the machined surface. Investigating several materials such as Garnet, NiZn and MgMn ferrite, Stern and Themme concluded that only magnetostrictive core materials are strongly affected by mechanical stress.

In 1970 John E. Knowles investigated how uniaxial stress affects the initial permeability - temperature curve, $\mu_{\mathrm{i}}(T)$, of $\mathrm{MnZn}$ ferrite with different $\mathrm{MnO} / \mathrm{ZnO}$ ratios [11]. Knowles compared the $\mu_{\mathrm{i}}(T)$ curve of sintered and ground toroid core samples and learned that the permeability of the machined toroids (abrasion with a belt grinder or sandpaper) was lowered at high temperatures and raised at lower temperatures resulting in a sharp bend in the $\mu_{\mathrm{i}}(T)$ curve and that the samples with higher $\mathrm{MnO} / \mathrm{ZnO}$-ratio were more sensitive. Knowles argued that the immense local pressure exerted during the grinding process caused a compressive stress in the surface layer and, consequently, a tensile stress in the interior of the ferrite. He further argued that it was hence possible for the compressed 
surface to influence the magnetic properties of the bulk ferrite. However, how much the properties of the bulk are affected depends on the exerted stress in the interior of the ferrite which is a function of geometry and size. In a follow-up publication in 1974, Knowles found that the loss factor, $\frac{\tan \delta}{\mu}$, of a ferrite pot core at $100 \mathrm{kHz}$ increased by up to $33 \%$ after machining the mating and air gap surfaces with a diamond-wheel precision grinding machine [12]. Knowles experimentally determined that the grinding process causes a very large residual compressive stress in the surface layer with magnitude of up to $700 \mathrm{MN} / \mathrm{m}^{2}$ (almost the ultimate compressive stress of the material) reducing almost linearly towards the interior of the sample, becoming negligible at a depth of $5 \mu \mathrm{m}$ below the surface. In another experiment, Knowles applied manually a pressure to both flat faces of a toroidal core in order to investigate the dependency of the loss factor, permeability and coercitive force on the compressive stress in the ferrite. Based on the results, Knowles estimated that the average loss factor of the ground surface is roughly 350 times larger than the intrinsic loss factor of the examined MnZn ferrite material. The magnetic properties of a ferrite rod subject to tensile and compressive stress were also studied by E. C. Snelling [13]. Snelling applied either a tensile or compressive stress and recorded the permeability, the residual and hysteresis loss factor and the coercitivity of the MnZn ferrite sample. In close agreement with Knowles, Snelling observed that above $30^{\circ} \mathrm{C}$ tension reduces the permeability and compression increases it and below about $-10^{\circ} \mathrm{C}$ the opposite is true. Snelling could also show that for both compressive and tensile stress, the residual and hysteresis loss factors increase.

Kloholm et al. determined the magnetostrictive response of thin MnZn and NiZn ferrite plates $(1 \mathrm{~cm} \times 4.2 \mathrm{~cm} \times 90 \mu \mathrm{m})$ as the applied magnetic field was decreased from saturation to magnetic remanence [14]. Additional abrasion of one side of the ferrite specimen with 180 grit $\mathrm{SiC}$ polishing paper caused a marked increase in magnetostrictive response of the sample, indicating that a deteriorated ferrite layer is formed underneath the machined surface. By knowing the magnetostriction constant of the investigated materials, Klokholm et al. estimated that the thickness of the deteriorated layer is about $40 \mu \mathrm{m}-50 \mu \mathrm{m}$ in case of the MnZn ferrite and around $90 \mu \mathrm{m}-100 \mu \mathrm{m}$ in the investigated NiZn ferrite.

The influence of machining on the properties of ferrite has also been studied extensively with respect to magnetic storage systems. NiZn and MnZn ferrite ceramics are widely used as recording head materials and are finished to a high degree of precision by diamond grinding and lapping. The finished ferrite surface is found to have a shallow magnetically inactive layer causing recording head performance to deteriorate [15]-[17].

For the sake of completeness it should furthermore be noted, that recently [18]-[20] reported that the cutting process has also a substantial influence on the ferromagnetic material properties of steel sheets used to manufacture laminated iron cores for electric machines.

\section{A. Microanalysis of MnZn Ferrite Surface}

From the review of the scientific literature it can be concluded that ferrite is extremely sensitive to mechanical stress and machining likely deteriorates the magnetic properties. In order to find structural evidence of a deteriorated layer close to the surface and assess the surface condition of the machined MnZn ferrite plates, a Scanning Electron Microscopy (SEM) analysis was performed and the results are depicted in Fig. 4. In Fig. 4(a) the surface with clearly visible individual grains of a sintered MnZn ferrite sample (3F4) is shown where no subsequent (i.e., after sintering) machining process was applied. In contrast, the ground surface as a result of cutting the plate from a long ferrite bar with a diamond saw is depicted in Fig. 4(b), showing deformed grains and grooves formed by the abrasive diamond particles. In order to assess the morphology of the ferrite underneath the surface, Focused Ion Beam (FIB) milling was performed as shown in Fig. 4(c) to expose the bulk ferrite material. The close-up view in Fig. 4(d) clearly indicates micro cavities and cracks roughly $5 \mu \mathrm{m}-10 \mu \mathrm{m}$ below the surface. Fig. 4(e) depicts the plate surface after removing roughly $500 \mu \mathrm{m}$ of material by means of abrasive polishing with $\mathrm{SiC}$ grinding paper and gradually decreasing the grain size from $22 \mu \mathrm{m}$ down to $5 \mu \mathrm{m}$, using water as a lubricant. The last polishing step was performed with colloidal silica suspension with a grain size of $60 \mathrm{~nm}$ and a soft polishing cloth. Fig. 4(f) depicts the lateral view (i.e. orthogonal to the machined surface) of the ferrite sample embedded in a Bakelite support after removing roughly $1 \mathrm{~mm}$ of material to expose the bulk. From Figs. 4(e) and (f) it becomes clear that the cavities and cracks identified in Fig. 4(c) are not caused by mechanical stress exerted during cutting, but are the result of imperfections in the sintering of the MnZn ferrite. It should be noted that this is not vendor but rather process or technology specific, since the same cavities and cracks were also visible in ferrite samples from other vendors.

However, the presented SEM microanalyses did not reveal any structural abnormality which would allow to actually pinpoint the excess core loss to shallow layers of ferrite with deteriorated magnetic performance as suggested in the literature. Therefore, an experimental approach is derived in the following which allows to differentiate between core losses associated with the bulk and the surface of the examined ferrite plates.

\section{Quantification of Surface Losses}

The nonlinear characteristics of magnetic core materials require large-signal measurements to determine core losses accurately. It is a common technique to experimentally measure core losses for sinusoidal excitation of different amplitudes and frequencies and then obtain the parameters of the well known Steinmetz Equation (SE),

$$
p=k f^{\alpha} \hat{B}^{\beta}\left(\mathrm{mW} / \mathrm{cm}^{3}\right),
$$

by means of least-mean-square regression. Using the SE with identified parameters $k, \alpha$ and $\beta$ then allows to accurately predict core losses for a limited flux density and frequency range.

Considering again a ferrite plate with cross section $A_{\mathrm{p}}$ and thickness $d_{\mathrm{p}}=2 d_{\mathrm{s}}+d_{\mathrm{b}}$ as shown in Fig. 3(b), where $d_{\mathrm{s}}$ and $d_{\mathrm{b}}$ are 


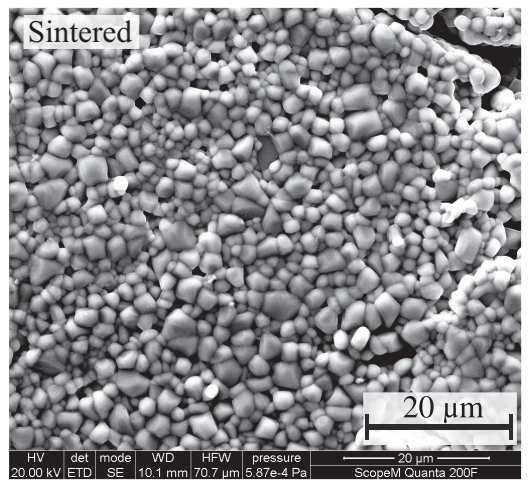

(a)

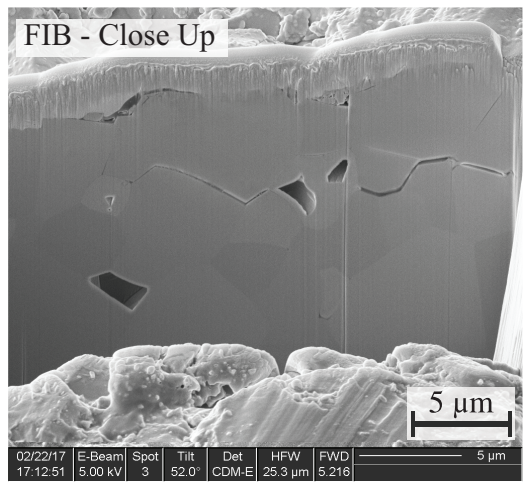

(d)

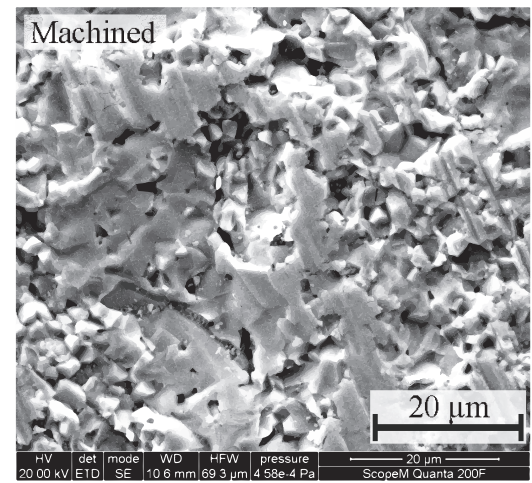

(b)

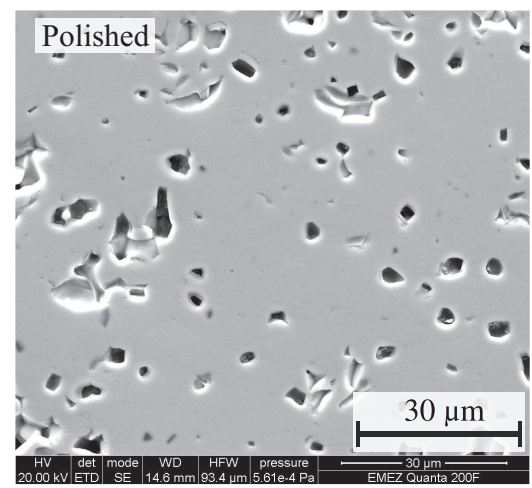

(e)

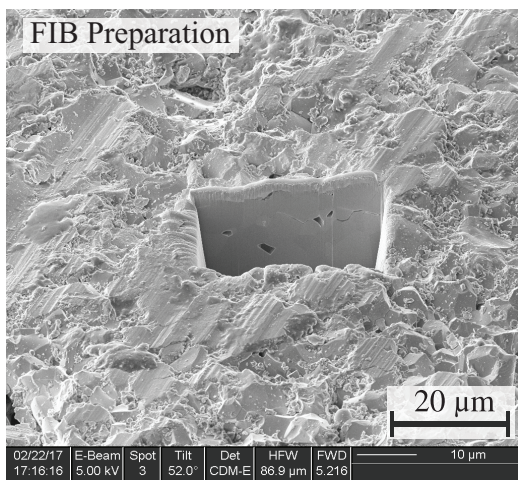

(c)

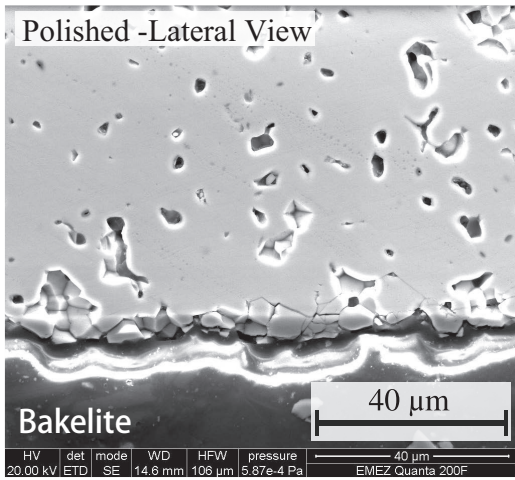

(f)

Fig. 4. Scanning Electron Microscopy (SEM) images of MnZn ferrite samples. (a) Surface of a ferrite plate after sintering (no machining). (b) Surface of a plate cut from a long ferrite bar with a diamond blade. (c) Focused Ion Beam (FIB) milling to expose bulk material underneath surface. (d) Close-up of the FIB prepared sample revealing cracks and cavities underneath surface. (e) Plate surface after removing roughly $500 \mu \mathrm{m}$ of ferrite by means of polishing, gradually reducing grain size of SiC paper and surface finish with colloidal silica suspension and soft cloth. (f) Lateral view of plate embedded in Bakelite support after polishing.

the thicknesses of the deteriorated layers at the plate surfaces and the bulk ferrite with intrinsic properties, respectively. To keep the derivation simple, it is assumed that there is a strict separation of surface layer and intrinsic material and that these segments exhibit a strictly cuboidal shape as indicated in Fig. 3(b). It then follows that for homogenous flux density, ensured by the excitation circuit described in Section III-B, the total core losses in the ferrite plate are given by

$$
\begin{aligned}
P_{1} & =\underbrace{k f^{\alpha} \hat{B}^{\beta}}_{\kappa} \underbrace{d_{\mathrm{b}} A_{\mathrm{p}}}_{\operatorname{Vol}_{b}}+\underbrace{k_{\mathrm{s}} f^{\alpha_{\mathrm{s}}} \hat{B}^{\beta \mathrm{s}}}_{\kappa_{\mathrm{s}}} \underbrace{2 d_{\mathrm{s}} A_{\mathrm{p}}}_{\operatorname{Vol}_{s}} \\
& =\left(\kappa \cdot d_{\mathrm{b}}+\kappa_{\mathrm{s}} 2 d_{\mathrm{s}}\right) A_{\mathrm{p}}=\left(\kappa+\kappa_{\mathrm{s}} \frac{2 d_{\mathrm{s}}}{d_{\mathrm{b}}}\right) d_{\mathrm{b}} A_{\mathrm{p}} \\
& \approx\left(\kappa+\kappa_{\mathrm{s}} \frac{2 d_{\mathrm{s}}}{d_{\mathrm{p}}}\right) d_{\mathrm{p}} A_{\mathrm{p}},
\end{aligned}
$$

wherein $d_{\mathrm{p}}=d_{\mathrm{b}}+2 d_{\mathrm{s}} \approx d_{\mathrm{b}}$ is the fair assumption that the thickness of the surface layer is negligible compared to the thickness of the bulk.

It is presented in the following, how a calorimetric measurement approach can be applied to experimentally determine both bulk and surface loss densities of a multi-gap core assembled from thin ferrite plates. The technique is based on determining core losses in ferrites by means of precise temperature rise monitoring as reported in [21]-[23].

A second experimental method based on electrical core loss measurements using ferrite samples with a distinct number of gaps is discussed in Appendix D. Since the calorimetric method fully excludes the loss of the test circuit required to adjust the magnetic excitation and therefore allows to capture the power loss in the sample directly, it features a significantly lower measurement error and is therefore the preferred choice in this study.

\section{A. Linear Thermal Model}

The power loss in the core given in (2) during continuous operation causes naturally a temperature rise of the ferrite plate over time which can be modeled by a lumped thermal network considering one-dimensional heat flow as shown in Fig. 5(a), wherein lumped parameters $C_{\mathrm{th}, \mathrm{s}}$ and $C_{\mathrm{th}, \mathrm{b}}$ capture the thermal capacitance of the surface layer and bulk material, respectively, and $R_{\mathrm{th}, \mathrm{b}-\mathrm{s}}$ models the virtual thermal resistance of the bulksurface interface. Since the loss density in bulk and surface layer is homogeneous and a plate features a comparably low thermal conductivity towards ambient at its thin lateral faces in $x, y$-direction, i.e., $R_{\mathrm{th}, \mathrm{b}-\mathrm{a}} \rightarrow \infty$, it is sufficient to consider a strict one-dimensional heat flow in pos. and neg. $z$-direction (i.e., normal to the plate surface; cf. Fig. 3(b)). The power loss in bulk and surface region is captured with distinct current sources 


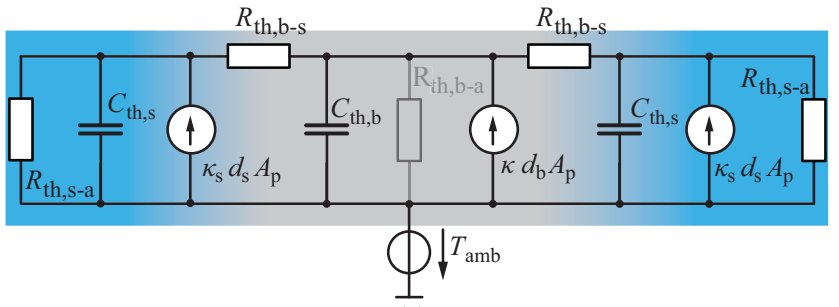

(a)

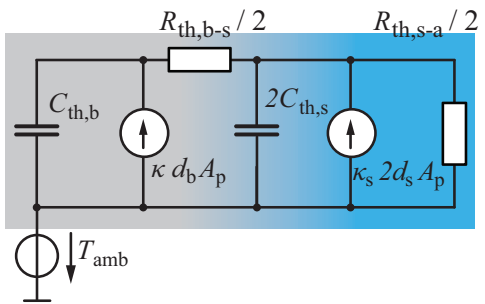

(b)

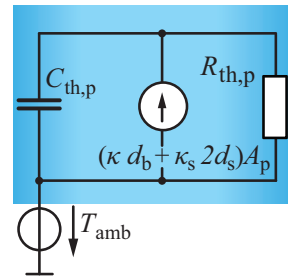

(c)

Fig. 5. (a) Thermal network of the ferrite plate considering one-dimensional heat flow in pos. and neg. z-direction (cf., Fig. 3(b)); lumped parameters $C_{\mathrm{th}, \mathrm{s}}$ and $C_{\mathrm{th}, \mathrm{b}}$ capture the thermal capacitance of the surface layer and bulk material, respectively, and $R_{\mathrm{thb} \text {-s }}$ models the thermal resistance of the bulk-surface interface. The power loss in bulk and surface region is captured with distinct current sources $\kappa d_{\mathrm{p}} A_{\mathrm{p}}$ and $\kappa_{\mathrm{s}} d_{\mathrm{s}} A_{\mathrm{p}}$, and the transfer of heat to ambient is taken into consideration with $R_{\mathrm{th}, \mathrm{s}-\mathrm{a}}$ and $R_{\mathrm{th}, \mathrm{b}-\mathrm{a}}$ (b) Simplification of the circuit due to symmetry and neglecting heat transfer from bulk to ambient $\left(R_{\mathrm{th}, \mathrm{b}-\mathrm{a}} \rightarrow \infty\right)$. (c) The thermal conductivity of ferrite is high enough to support a homogenous temperature distribution within the plate, further simplifying the thermal network.

TABLE II

Technical Details and Material Parameter

\begin{tabular}{|c|c|c|c|}
\hline Param. & Value & Unit & Description \\
\hline$A_{\mathrm{p}}$ & $7 \times 6.4$ & $\mathrm{~mm}^{2}$ & Cross section area of plate / sample \\
\hline$\lambda_{\mathrm{m}}$ & 0.154 & $\mathrm{~W} /(\mathrm{m} \mathrm{K})$ & Thermal conductivity of Mylar \\
\hline$\lambda_{\mathrm{fe}}$ & $3.5-5$ & $\mathrm{~W} /(\mathrm{m} \mathrm{K})$ & Thermal conductivity of MnZn ferrite \\
\hline$R_{\mathrm{th}, \mathrm{b}}$ & $13-20$ & $\mathrm{~K} / \mathrm{W}$ & Thermal resistance of a $3 \mathrm{~mm} \mathrm{MnZn}$ ferrite plate \\
\hline$R_{\mathrm{th}, \mathrm{m}}$ & 14.9 & $\mathrm{~K} / \mathrm{W}$ & Thermal resistance of a $100 \mu \mathrm{m}$ Mylar foil \\
\hline$C_{\mathrm{th}, \mathrm{p}}$ & 0.51 & $\mathrm{~J} / \mathrm{K}$ & $\begin{array}{l}\text { Thermal capacitance of a } 3 \mathrm{~mm} \mathrm{MnZn} \text { ferrite plate calculated } \\
\text { with specific heat capacity }\end{array}$ \\
\hline$C_{\mathrm{th}, \mathrm{m}}$ & 7.3 & $\mathrm{~mJ} / \mathrm{K}$ & Thermal capacitance of $100 \mu \mathrm{m}$ Mylar foil \\
\hline$c_{\mathrm{m}}$ & 1172 & $\mathrm{~J} /(\mathrm{kg} \mathrm{K})$ & Specific heat capacity of Mylar \\
\hline$c_{\mathrm{fe}}$ & 800 & $\mathrm{~J} /(\mathrm{kg} \mathrm{K})$ & Specific heat capacity of MnZn ferrite \\
\hline$\rho_{\mathrm{fe}}$ & 4800 & $\mathrm{~kg} / \mathrm{m}^{3}$ & Density of MnZn ferrite (3F45) \\
\hline$\rho_{\mathrm{m}}$ & 1390 & $\mathrm{~kg} / \mathrm{m}^{3}$ & Density of Mylar \\
\hline$R_{\text {th, a }}$ & 117.8 & $\mathrm{~K} / \mathrm{W}$ & Thermal resistance of $3 \mathrm{~mm}$ poly carbonate $(\mathrm{PC})$ air-gap lattice \\
\hline$R_{\text {th }}$ & 37.8 & $\mathrm{~K} / \mathrm{W}$ & Equivalent thermal resistance from calibration (cf., Fig. 11(b)) \\
\hline$C_{\text {th }}$ & 3.83 & $\mathrm{~J} / \mathrm{K}$ & Equivalent thermal capacitance from calibration (cf., Fig. 11(b)) \\
\hline
\end{tabular}

(cf., $\kappa d_{\mathrm{b}} A_{\mathrm{p}}$ and $\kappa_{\mathrm{s}} d_{\mathrm{s}} A_{\mathrm{p}}$ ) and a heat transfer to ambient in z-direction is taken into consideration with $R_{\mathrm{th}, \mathrm{s}-\mathrm{a}}$. Due to symmetry, the network can be simplified as shown in Fig. 5(b). As will become evident from the thermography images provided in Section III-B, the thermal conductivity of the MnZn-ferrite (cf., Table II) is large enough to support a homogenous temperature distribution within the plate, i.e., $R_{\mathrm{th}, \mathrm{b}-\mathrm{s}} \rightarrow 0$.

Accordingly, it is sufficient to consider the simplified first order thermal network with lumped parameters $R_{\mathrm{th}, \mathrm{p}}$ and $C_{\mathrm{th}, \mathrm{p}}$ as shown in Fig. 5(c), where the total dissipated power is combined in a single current source. It follows from (2) that the average loss density in a plate of thickness $d_{\mathrm{p}}$ is given by

$$
\bar{\kappa}=P_{1} / d_{\mathrm{p}} A_{\mathrm{p}}=\kappa+\kappa_{\mathrm{s}} 2 d_{\mathrm{s}} / d_{\mathrm{p}} .
$$

Thus, based on the hypothesis that the loss density is larger in the surface layer than in the bulk, the average loss density increases inverse proportional with the thickness of the plate. As illustrated in Fig. 6(a), a thin plate $\left(d_{1}\right)$ is more governed by the characteristics of the surface layer and features a higher avg. loss density as opposed to a fairly thick plate $\left(d_{3}\right)$ which approaches the loss density of the bulk ferrite.

If the heat transfer to ambient is negligible by means of sufficient thermal insulation (i.e., $R_{\mathrm{th}, \mathrm{p}} \rightarrow \infty$ in Fig. 6(a)), the temperature of the plate increases linearly over time,

$$
T(t)=\frac{P_{1}}{C_{\mathrm{th}, \mathrm{p}}} \cdot t+T_{\mathrm{amb}},
$$

where it is assumed that the initial temperature of the ferrite plate at $t=0$ equals the ambient temperature, $T_{\mathrm{amb}}$. Inserting expression (2) in (4) yields

$$
T(t)=\left(\kappa+\kappa_{\mathrm{s}} \frac{2 d_{\mathrm{s}}}{d_{\mathrm{p}}}\right) \frac{d_{\mathrm{p}} A_{\mathrm{p}}}{C_{\mathrm{th}, \mathrm{p}}} t+T_{\mathrm{amb}} .
$$

The equivalent thermal capacitance of the plate, $C_{\text {th,p }}$, can be determined from a calibration measurement as described later in Section III-E or calculated according to

$$
\begin{aligned}
C_{\mathrm{th}, \mathrm{p}} & =\left(c_{\mathrm{f}, \mathrm{s}} \rho_{\mathrm{f}, \mathrm{s}} 2 d_{\mathrm{s}}+c_{\mathrm{f}} \rho_{\mathrm{f}} d_{\mathrm{b}}\right) A_{\mathrm{p}} \\
& \approx c_{\mathrm{f}} \rho_{\mathrm{f}} d_{\mathrm{p}} A_{\mathrm{p}}=\tilde{c}_{\mathrm{f}} d_{\mathrm{p}} A_{\mathrm{p}},
\end{aligned}
$$

where $c_{\mathrm{f}}$ and $\rho_{\mathrm{f}}$ is the specific heat capacity and density of $\mathrm{MnZn}$ ferrite as listed in Table II, respectively. Note that it is assumed that the thermal capacitance of the plate is predominantly governed by the bulk properties. Substituting (6) in (5) leads to

$$
T(t)=\left(\frac{\kappa}{\tilde{c}_{\mathrm{f}}}+\frac{\kappa_{\mathrm{s}}}{\tilde{c}_{\mathrm{f}}} \frac{2 d_{\mathrm{s}}}{d_{\mathrm{p}}}\right) t+T_{\mathrm{amb}} .
$$




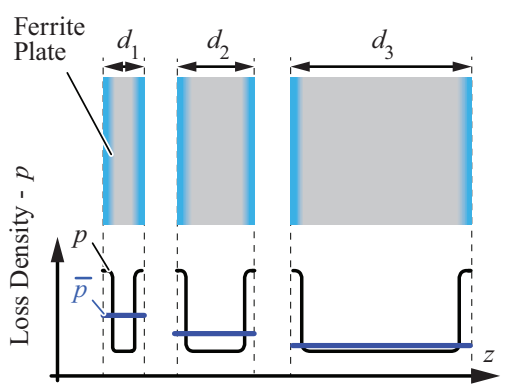

(a)

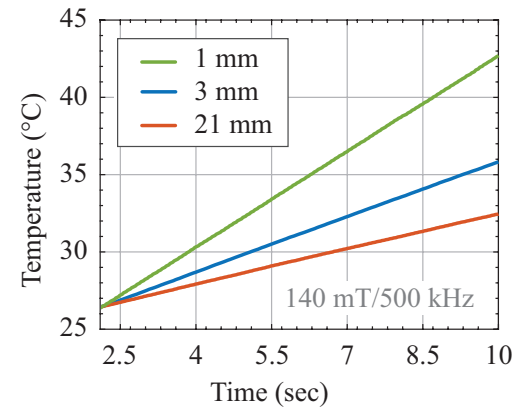

(b)

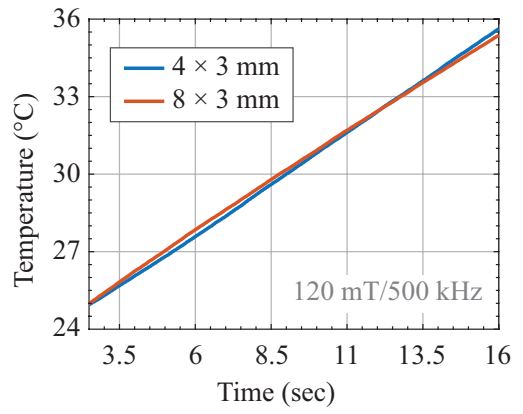

(c)

Fig. 6. (a) A thin plate $\left(d_{1}\right)$ is more governed by the characteristics of the surface layer and features a higher avg. loss density, $\bar{p}$, as opposed to a fairly thick plate $\left(d_{3}\right)$ which approaches the loss density of the bulk ferrite. (b) Experimental temperature rise measurement showing that a sample assembled from 1 mm plates results in a much steeper slope compared to $3 \mathrm{~mm}$ plates or the single-piece $(21 \mathrm{~mm})$ reference sample. (c) Experimental measurement showing that the slope of the temperature rise is not affected by the actual height of the stack (i.e., number of plates in the stack).

It can be seen that the temperature rise is governed by a constant contribution of the bulk material and a contribution of the power dissipation in the surface layer which depends on the actual ratio of surface to plate thickness. This trend is strongly supported by the experimental results depicted in Fig. 6(b), where it can be seen that a multi-gap sample assembled from thin $1 \mathrm{~mm}$ plates features a much steeper slope in contrast to thicker $3 \mathrm{~mm}$ plates and the (very thick) solid reference sample. Interestingly, since both total power loss and total thermal capacitance increases proportional to the number of stacked plates, the slope of the temperature rise is not affected by the actual height of the stack (i.e., number of plates in the stack) as confirmed by the measurement results depicted Fig. 6(c). This in turn allows to measure a stack rather than a single plate which is advantageous in accommodating the required flux sense winding and improves mechanical handling of the sample for a precise placement in the test circuit (cf., Section III-B).

Since the properties of ferrite are strongly depending on temperature, the duration $t_{x}$ to reach a specified temperature difference, $\Delta T=T_{\text {end }}-T_{\text {amb }}$, is measured rather than a final temperature after a fixed time span $\Delta t$. Rearranging (7) leads to

$$
t_{\chi}=\frac{\Delta T}{\frac{\kappa}{\tilde{c}_{\mathrm{f}}}+\frac{\kappa_{\mathrm{s}}}{\tilde{c}_{\mathrm{f}}} \frac{2 d_{\mathrm{s}}}{d_{\mathrm{p} x}}}
$$

for a plate with thickness $d_{\mathrm{px}}$. Now, performing two consecutive measurements with identical operating point but using ferrite plates with different thicknesses, $d_{\mathrm{p} 1}$ and $d_{\mathrm{p} 2}$, allows to solve (8) to obtain the loss density of the bulk ferrite,

$$
\kappa=\frac{\left(d_{\mathrm{p} 1} t_{2}-d_{\mathrm{p} 2} t_{1}\right)}{\left(d_{\mathrm{p} 1}-d_{\mathrm{p} 2}\right) t_{1} t_{2}} \tilde{c}_{\mathrm{f}} \Delta T,
$$

and the loss per surface area,

$$
\tilde{\kappa}_{\mathrm{s}}=\kappa_{\mathrm{s}} d_{\mathrm{s}}=\frac{1}{2} \frac{d_{\mathrm{p} 1} d_{\mathrm{p} 2}\left(t_{1}-t_{2}\right)}{\left(d_{\mathrm{p} 1}-d_{\mathrm{p} 2}\right) t_{1} t_{2}} \tilde{c}_{\mathrm{f}} \Delta T .
$$

Note that since the actual thickness of the surface layer $d_{\mathrm{s}}$ is unknown, it is more suitable to define power loss per surface area, $\left[\tilde{\kappa}_{\mathrm{s}}\right]=\mathrm{mW} / \mathrm{cm}^{2}$.

\section{B. Experimental Setup}

An excitation circuit is needed which ensures a homogeneous flux density distribution in the sample, allows to characterize the core loss for different flux density amplitudes and frequencies, and facilitates an easy installation of different samples. A magnetic circuit resembling an E-type core with the multigap sample located in the center limb as depicted in Fig. 7(a) is chosen in this study, since it only requires a single sample as opposed to a U-type setup and can be assembled by individual off-the-shelf available ferrite cuboids minimizing custom machining effort. The multi-gap sample in the center as well as the individual ferrite cuboids are fixated by means of a 3D printed mounting fixture and fastening screws. Consistent pressure applied to the ferrite regardless of the inserted sample is guaranteed by means of a torque wrench adjusted to $0.02 \mathrm{Nm}$. In Fig. 7(c), two multi-gap samples assembled with $1 \mathrm{~mm}$ and $3 \mathrm{~mm}$ thick plates, respectively, and a solid, single piece ferrite sample are shown. The equivalent circuit of the test setup is shown in Fig. 8, where the transformer equivalent circuit with open secondary winding represents the magnetic excitation circuit with flux sense winding. The reactive power of the magnetic circuit is compensated by means of a series connected capacitor in order to reduce the burden on the HF voltage source (Iwatsu IE-1125B) and attain all operating points $\{\hat{B}, f\}$ of interest given the output voltage limitation of the amplifier.

Since the outer magnetic circuit contributes to the total core losses (troublesome in the case of the electrical loss measurement, cf., Appendix D) and heat generation in the test circuit can affect sample temperature, the dimensions of the outer magnetic circuit are chosen such that its losses are minimized while keeping the flux density in the sample homogeneous. This can be achieved by increasing the cross section of the magnetic path while minimizing its avg. magnetic length, since the sample-to-core loss ratio considering homogenous flux density in the setup is given by

$$
\frac{P_{\text {sample }}}{P_{\text {test }}} \propto\left(\frac{A_{\text {test }}}{A_{\mathrm{p}}}\right)^{\beta-1} \frac{l_{\mathrm{m}, \text { sample }}}{l_{\mathrm{m}, \text { test }}},
$$




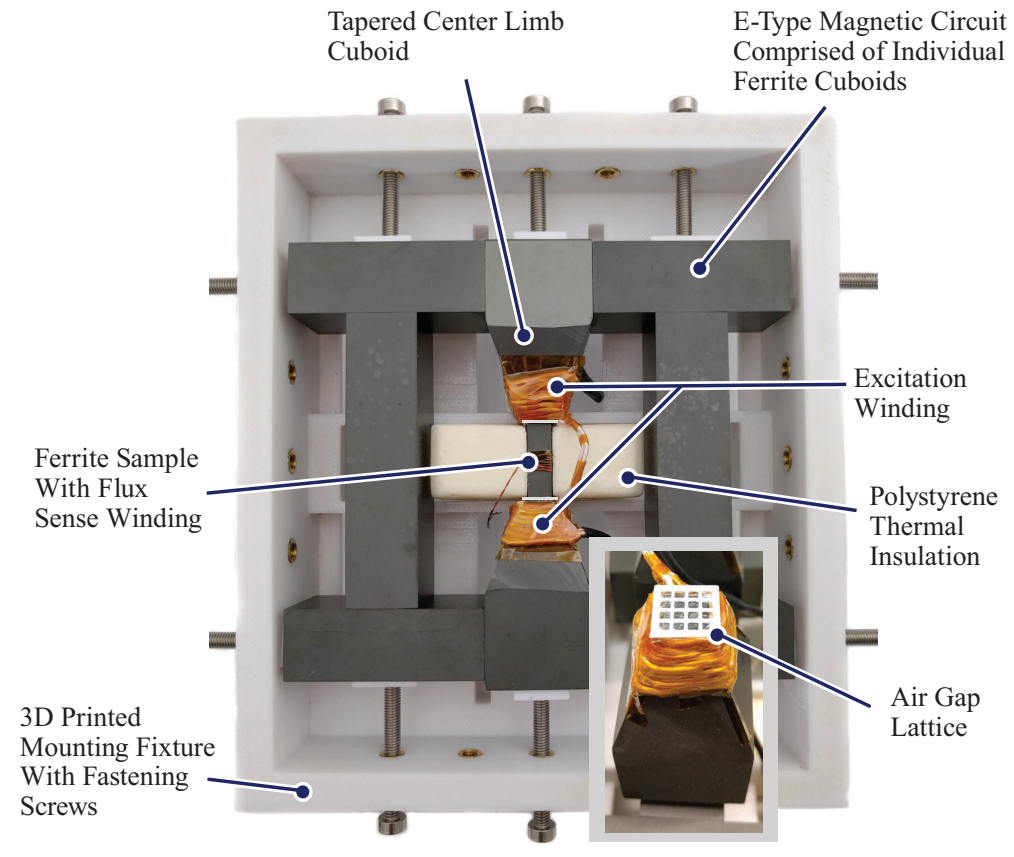

(a)

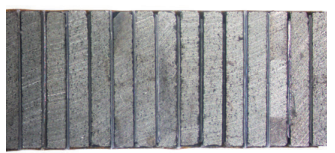

Stack of $1 \mathrm{~mm}$ Plates

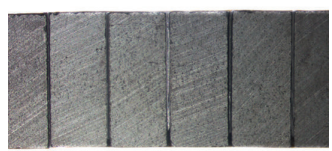

Stack of 3 mm Plates

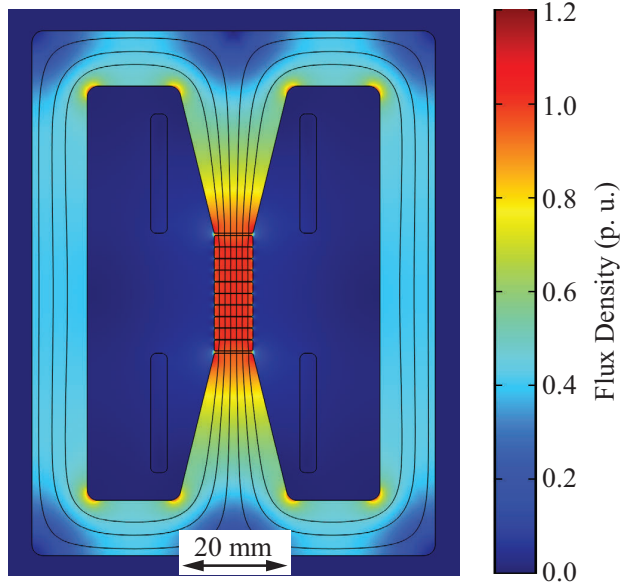

(b)

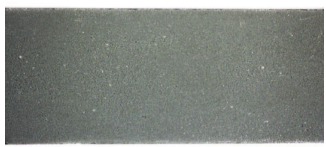

Solid Sample (Single Piece)

(c)

Fig. 7. (a) Test circuit resembling an E-type core with a multi-gap core sample located in the middle of the center limb; fastening screws and a 3D printed mounting fixture keeping the individual ferrite cuboids of the outer magnetic circuit and the sample in position; splitting the turns of the excitation winding in two sections above and below the sample and employing tapered center limbs to accommodate the cross section difference between outer magnetic circuit and sample facilitates a homogenous flux density distribution in the sample; a dedicated tightly wound sense winding is placed around the center of the sample to correctly adjust the peak flux density; measures to increase the thermal resistance to ambient are the polystyrene casing and the air gap lattice inserted between sample and test circuit. (b) FEM simulation result showing the homogenous flux density distribution in the sample. (c) Picture of multi-gap samples composed of 1 mm and 3 mm plates, respectively, and the single piece solid reference sample. To adjust the partial gap length, $100 \mu \mathrm{m}$ thick Mylar foil is inserted between the plates.

where $A_{\text {test }}, A_{\mathrm{p}}$ and $l_{\mathrm{m}, \text { test }}, l_{\mathrm{m}, \text { sample }}$ denote the cross section area and avg. magnetic path length of the test circuit and sample, respectively. However, due to the distributed gap, the comparably large magnetic reluctance of the installed multigap sample in the center limb results in a significant leakage flux which potentially causes an inhomogeneous flux density distribution in the sample or bypasses it completely and only contributes to undesired loss in the test circuit. With the aid of magnetostatic field simulations (cf., Fig. 7(b)), optimal dimensions based on readily available core shapes were obtained which maximize the sample-to-core loss ratio of the setup while keeping the flux density distribution in the sample homogenous. As shown in Fig. 7(a), two measures were employed which greatly contribute to a homogenous flux density distribution in the sample: (i) the turns of the excitation winding are split in two sections directly above and below the sample and (ii) tapered center limbs are employed to accommodate the cross section difference between test circuit and sample. In order to correctly adjust the desired flux density amplitude in the multi-gap sample despite different cross section areas and leakage flux, a dedicated tightly wound sense winding is placed around the center of the sample as indicated in Fig. 7(a).

As mentioned previously in Section III-A, due to the constant loss density and the low thermal conductivity towards ambient in lateral direction, a uniform temperature distribution in $(x, y)$ direction (cf., Fig. 3) is present throughout the sample which allows to infer the correct temperature from a measurement at one of the lateral surfaces. In this paper, infrared thermography is the preferred temperature instrumentation since (i) it allows to compute the average temperature over the entire multi-gap sample as opposed to a single spot measurement provided by fiber optical temperature probes, thermistors (NTC, PTC) or thermocouples and (ii) is contactless, i.e., does not require to physically attach a probe to the delicate sample. Moreover, infrared thermography reveals non-uniform temperature distributions and the formation of hot spots and thus allows to detect inhomogeneous flux density distribution in the sample (f.i. flux crowding) caused by a potential sample misalignment and/ or an excessive gap between sample and center limb pole pieces 


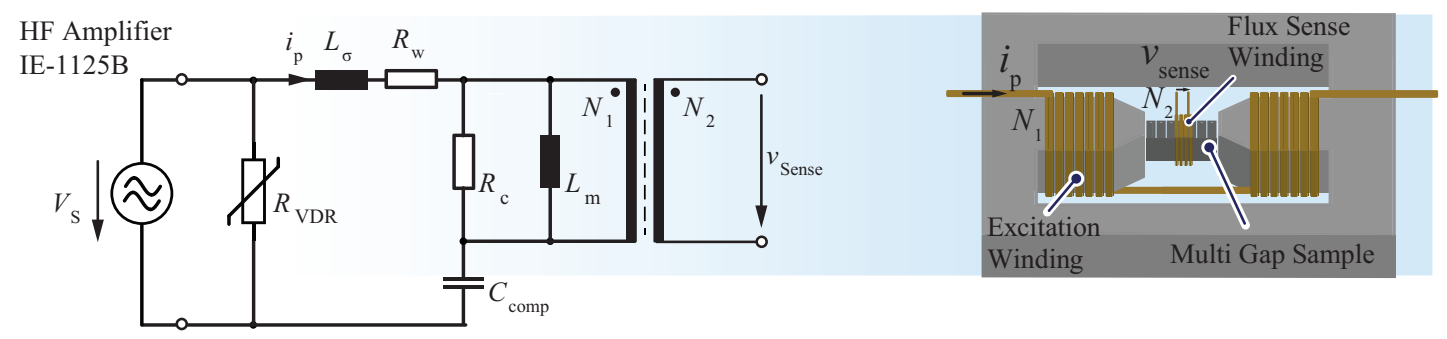

Fig. 8. Schematic of the employed excitation circuit; the dedicated sense winding with $N_{2}$ turns allows to precisely determine the flux density. The reactive power of the magnetic circuit is compensated by means of a capacitor bank to reduce the burden on the HF voltage source and attain all operating points $\{\hat{B}, f\}$ of interest despite the limited HF amplifier voltage range.

of the test circuit. Using thermography to determine core loss distribution in magnetic components has been reported in [24], [25] and to detect defects in ferromagnetic laminated sheets and soft mangetic composites in [26]. The authors in [27], applied infrared thermography to visualize the iron loss distribution in a permanent magnet motor. A picture of the experimental measurement setup is depicted in Fig. 9(a), showing the implemented test setup according to Fig. 8 with the magnetic circuit and capacitor bank and a FLIR A655sc high definition infrared camera pointed towards the multi-gap sample. The camera is equipped with additional close-up lens to allow a working distance of around $6 \mathrm{~cm}-7 \mathrm{~cm}$ between sample and camera lens and provides $50 \mu \mathrm{m}$ spatial resolution. The lower $\pm 2{ }^{\circ} \mathrm{C}$ absolute measurement accuracy of a microbolometer [28] is not relevant here, since according to (9) and (10) only a temperature difference must be determined and the standard deviation per detector pixel of a high end infrared camera is typical around $0.1{ }^{\circ} \mathrm{C}-0.2{ }^{\circ} \mathrm{C}$ [29]. Compensating the reactance of the magnetic test circuit is necessary to attain all $\{\hat{B}, f\}$ operating points of interest given the limited voltage range of the employed IWATSU IE-1125B HF power amplifier. For a good agreement with the linear model derived in Section III-A, the heat transfer from sample to ambient in the experimental setup must be reduced as much as possible. To prevent convective heat transfer from the lateral surfaces of each individual plate in the stack to ambient, the sample is encased in polystyrene except for the surface pointing towards the infrared camera as it can be seen from Fig. 7(a) and Fig. 9(a). Furthermore, to minimize conductive heat transfer from the multi-gap sample to the two center limb core pieces of the E-type test circuit, an additional gap between sample and adjacent pole pieces is inserted. A gap length of around $0.5 \mathrm{~mm}$ is a still acceptable trade-off between minimizing conductive heat transfer and establishing a homogenous flux density in the sample close to the interfaces. In order to achieve the lowest possible thermal conductivity, the gap is realized by means of a 3D printed lattice made out of polycarbonate as it is depicted in the detail view of Fig. 7(a).

The experimental results presented in Section IV of this paper were obtained by relating the slope of the temperature rise of a multi-gap sample with 7 stacked $3 \mathrm{~mm}$ ferrite plates $\left(d_{\mathrm{p} 1}=3 \mathrm{~mm}\right)$ to a solid, single-piece reference sample of $21 \mathrm{~mm}$ length $\left(d_{\mathrm{p} 2}=21 \mathrm{~mm}\right)$. Mylar foil is inserted between adjacent plates to establish a gap length of $100 \mu \mathrm{m}$, which,
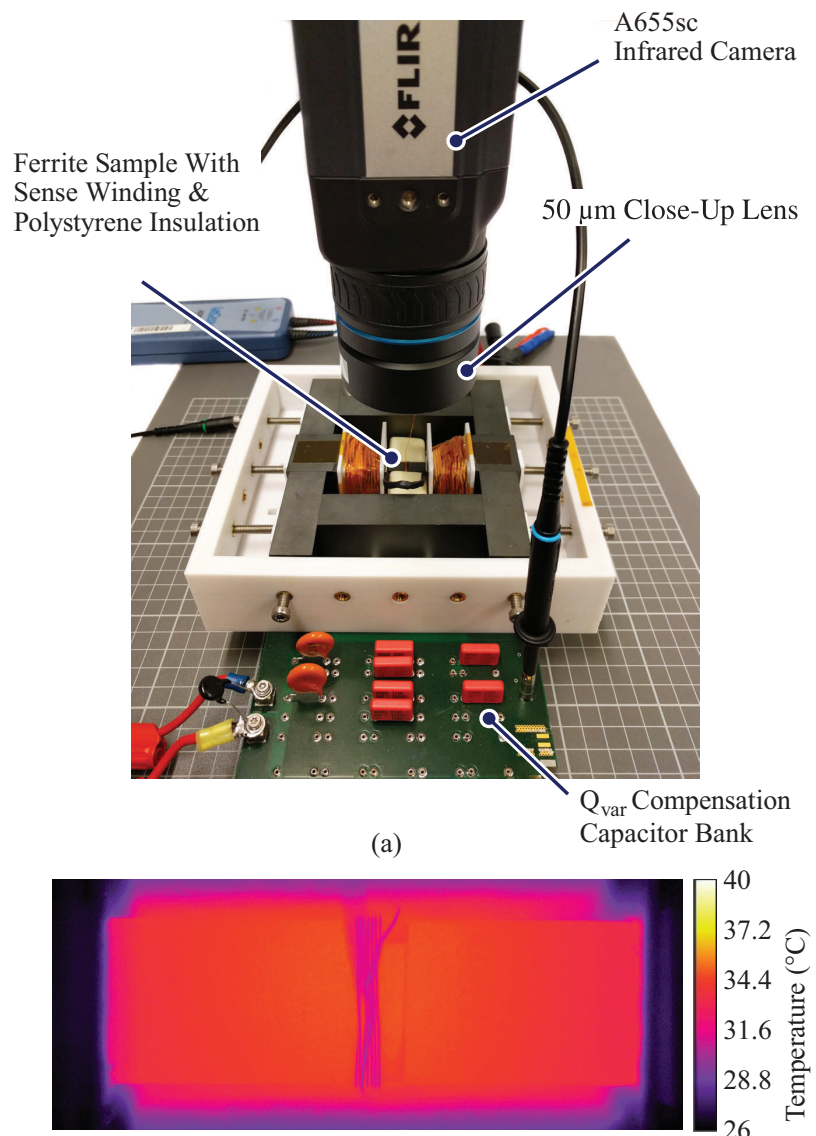

(b)

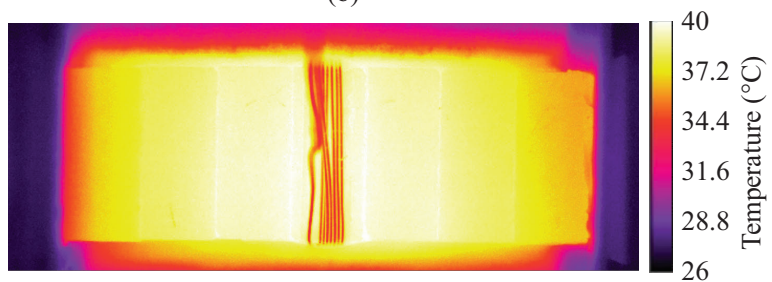

(c)

Fig. 9. (a) Picture of the experimental setup showing the magnetic test circuit with capacitor bank for reactive power compensation and the employed FLIR A655sc high definition infrared camera pointed towards the sample. Thermography image of the temperature rise of the solid (b) and multi-gap sample (c) subject to an excitation with $125 \mathrm{mT}$ at $400 \mathrm{kHz}$ exactly after $30 \mathrm{~s}$ of total elapsed measurement time. It is clearly visible that the multi-gap sample composed of $7 \times 3 \mathrm{~mm}$ plates is heating up much quicker.

in combination with $3 \mathrm{~mm}$ plates, completely alleviates loss 
contribution arising from flux crowding in the sample caused by assembly and mechanical tolerances as described in Appendix A.

At each operating point of interest, $\{\hat{B}, f\}$, the temperature rise after abruptly enabling the correctly adjusted power amplifier is recorded for both samples and the core and surface loss density are then computed according to (9) and (10). The elapsed time to reach a specific temperature difference $\Delta T$ starting from ambient temperature is extracted from the captured temperature rise in a subsequent post-processing step. Since the core loss and many other properties of ferrite depend strongly on operating temperature, a large temperature deflection would certainly have an impact on the results. However, the high frame rate of the infrared camera (up to $50 \mathrm{~Hz}$ with full resolution of $640 \times 480$ ) allows to determine the elapsed time even for a very small temperature difference of just a few ${ }^{\circ} \mathrm{C}$. The experimental results presented in this paper were obtained for $\Delta T=2.5^{\circ} \mathrm{C}$.

A snapshot of the temperature rise of the solid and multigap sample subjected to an excitation with $125 \mathrm{mT}$ at $400 \mathrm{kHz}$ exactly after $30 \mathrm{~s}$ of total elapsed measurement time is shown in Fig. 9(b) and (c), respectively. Since both measurements were started from ambient temperature, $T_{\text {amb }}=26.4{ }^{\circ} \mathrm{C}$, it can be clearly seen that the multi-gap sample heats up much faster showing a final temperature reading of around $38{ }^{\circ} \mathrm{C}$ in contrast to roughly $32{ }^{\circ} \mathrm{C}$ of the solid sample. Note that the interruption of the uniform temperature distribution by the sense winding is in the center of each sample.

\section{Longitudinal Temperature Gradient}

Referring to Fig. 9(b) and (c), it is noticeable that both samples exhibit a temperature gradient in longitudinal direction with slightly lower temperatures at both ends where the sample connects to the outer magnetic circuit. Fig. 10 depicts the computed temperature profile of both infrared images in longitudinal direction, whereby the temperature reading at every camera pixel in the plot represents the mean temperature of all sensor pixels encompassing the sample in transversal direction (i.e., orthogonal to the direction of the flux). It becomes evident that the temperature gradient in the multi-gap sample is more pronounced $\left(\approx 3.75^{\circ} \mathrm{C}\right)$ compared to the solid reference sample $\left(\approx 1.25^{\circ} \mathrm{C}\right)$. Moreover, the temperature profile of the multi-gap sample exhibits a clear local maximum whenever two surfaces of adjacent ferrite plates meet, i.e., at the surfaces of the plates. Now, a representative thermal network of stacked plates is shown in Fig. 11(a) which is helpful to explain the more pronounced temperature gradient in the case of the multi-gap sample. Note that the simplified thermal model of a single plate according to Fig. 5(c) is considered. Since the thermal capacitance of a piece of Mylar foil is negligible compared to the ferrite plate (cf., Table II) only the thermal resistance introduced by the gap material, $R_{\mathrm{th}, \mathrm{m}}$, is included in the model. Although the thermal conductivity of Mylar is at least a factor 10 lower compared to $\mathrm{MnZn}$ ferrite, the resulting thermal resistance of foil and ferrite plate is in the same order of magnitude $(\approx 15 \mathrm{~K} / \mathrm{W}$ as listed in Table II). However, since the length of both samples examined in this study is equal per design and the Mylar foil has virtually no contribution,

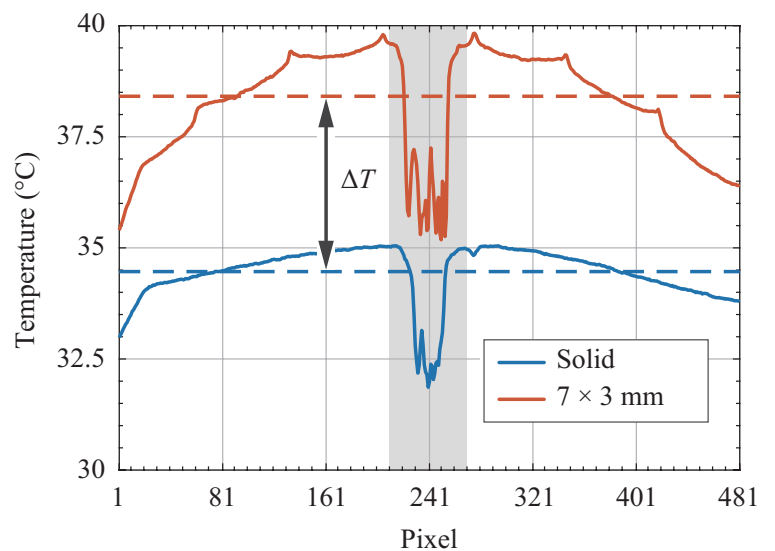

Fig. 10. Temperature profile in longitudinal direction of the IR images of solid and multi-gap sample shown in Fig. 9 (b) and (c); the dashed line represents the average value of the IR sensor and is used to compute $\Delta T$.

the effective thermal capacitance of both solid and multi-gap sample is identical. Ideally, if $R_{\mathrm{th}, \mathrm{a}} \rightarrow \infty$ then each plate will exhibit exactly the same temperature rise irrespective of the actual position in the stack as confirmed by a circuit simulation. However, the thermal resistance achieved with the air gap lattice is estimated to be around $R_{\mathrm{th}, \mathrm{a}}=117.8 \mathrm{~K} / \mathrm{W}$ considering the thermal conductivity of polycarbonate (PC) and air (air gap lattice, cf., Fig. 7(a)), and evidently not large enough to support uniform heating in longitudinal direction. It follows that the temperature gradient is more pronounced in the case of the stacked plate assembly since heat close to the center of the sample encounters an effectively larger thermal resistance in longitudinal direction towards both ends of the sample in comparison to the solid, single-piece sample. Now, in order to determine the elapsed time to reach a specific temperature difference, $\Delta T$, the pragmatic way of averaging the IR sensor reading also in the longitudinal direction is followed as indicated with dashed lines in Fig. 10, naturally excluding the distortion in the center of the sample caused by the flux sense winding. The corresponding first order thermal model is shown in Fig. 11(b) and the expression for the core and surface loss densities based on the linear model are refined in the following. Note that in the following, $R_{\mathrm{th}}$ and $C_{\mathrm{th}}$ denote the equivalent thermal parameters of a stack of plates (cf., $R_{\mathrm{th}, \mathrm{p}}$ and $C_{\mathrm{th}, \mathrm{p}}$ of a single plate, Fig. 5(c)).

\section{Exponential Thermal Model for Multi-Gap Assembly}

Based on the equivalent circuit shown in Fig. 11(b), the temperature rise of an assembly with $m$ plates of thickness $d_{\mathrm{p}}$ starting from $T_{\text {amb }}$ at $t=0$ is given by

$$
\begin{aligned}
T(t) & =P_{1 \Sigma} R_{\mathrm{th}}\left(1-e^{-\frac{t}{\tau}}\right)+T_{\mathrm{amb}} \\
& =\left(\kappa+\kappa_{\mathrm{s}} 2 d_{\mathrm{s}} / d_{\mathrm{p}}\right) m V_{\mathrm{p}} R_{\mathrm{th}}\left(1-e^{-\frac{t}{\tau}}\right)+T_{\mathrm{amb}},
\end{aligned}
$$

with the time constant $\tau=R_{\mathrm{th}} C_{\mathrm{th}}$ and the core volume of a plate $V_{\mathrm{p}}=d_{\mathrm{p}} A_{\mathrm{p}}$. It should be emphasized, that the total core volume $V_{\mathrm{p} \Sigma}=m V_{\mathrm{p}}$ is kept constant by adopting the plate thickness in accordance to the number of gaps such that the examined 


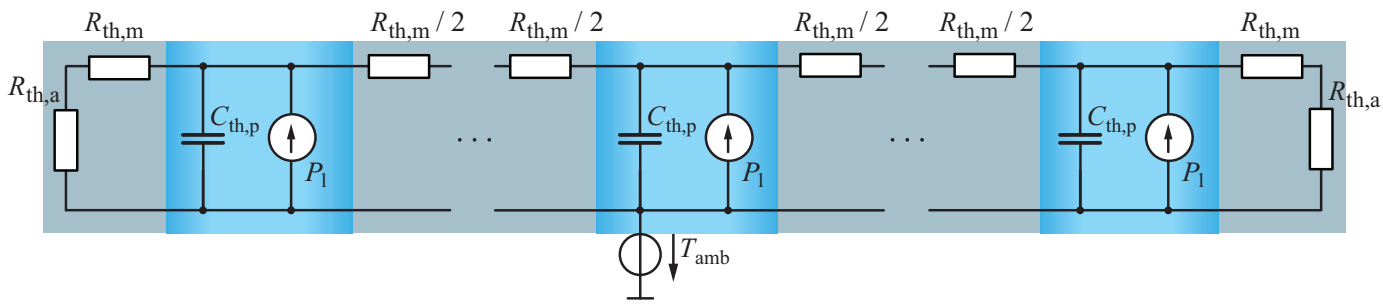

(a)

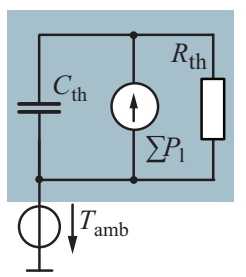

(b)

Fig. 11. (a) Representative thermal network of stacked plates using the simplified thermal model of a single plate according to Fig. 5(c) and considering the thermal resistance $R_{\mathrm{th}, \mathrm{m}}$ introduced by the gap material. (b) First order thermal model of a sample corresponding to averaged IR temperature reading in longitudinal direction.

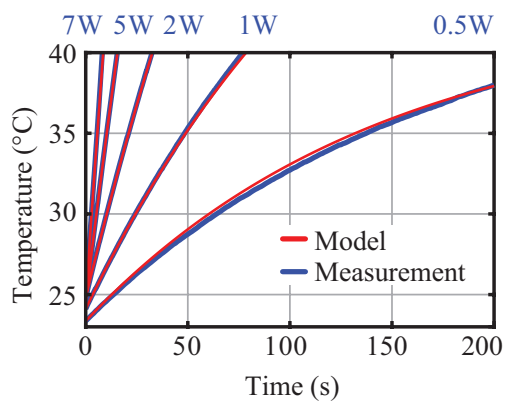

(a)

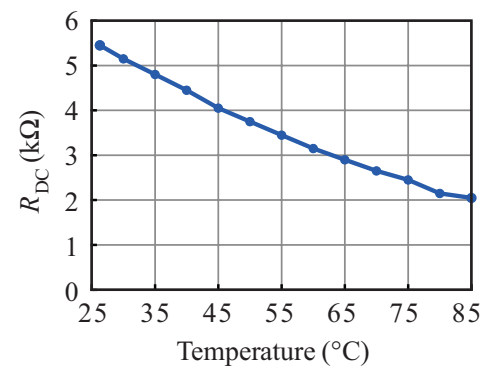

(b)

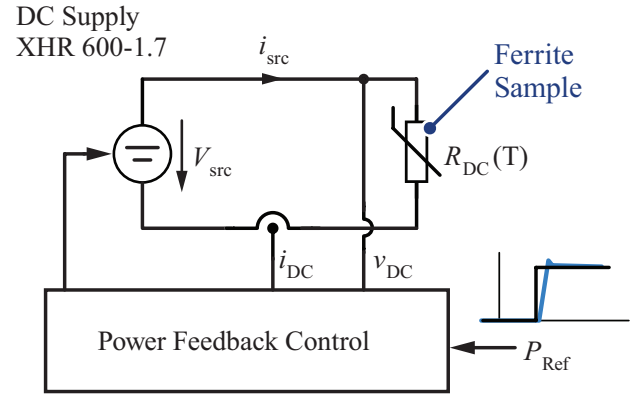

(c)

Fig. 12. (a) Measured temperature response obtained for the solid sample (blue) and the simulated response of the mathematical model with identified parameters (red) for several impressed power levels ranging from $0.5 \mathrm{~W}$ to $7 \mathrm{~W}$. (b) Measured DC resistance of MnZn ferrite as a function of temperature. (c) Schematic of implemented power feedback control to impress constant power during calibration despite temperature dependent change of the ferrite DC resistance.

multi-gap samples feature equal thermal parameters $R_{\mathrm{th}}, C_{\mathrm{th}}$. Analogously to the derivation described in Section III-A, measuring the elapsed time $t_{1}$ and $t_{2}$ of two samples with distinct plate thickness $d_{\mathrm{p} 1}$ and $d_{\mathrm{p} 2}$ to reach a specific $\Delta T$ allows to calculate the core loss density

$$
\kappa=\frac{d_{\mathrm{p} 1}\left(1-e^{-\frac{t 2}{\tau}}\right)-d_{\mathrm{p} 2}\left(1-e^{-\frac{t 1}{\tau}}\right)}{\left(d_{\mathrm{p} 1}-d_{\mathrm{p} 2}\right)\left(1-e^{-\frac{t 1}{\tau}}\right)\left(1-e^{-\frac{t 2}{\tau}}\right)} \frac{\Delta T}{R_{\mathrm{th}} V_{\mathrm{p} \Sigma}},
$$

and the surface loss density

$$
\tilde{\kappa}_{\mathrm{s}}=\frac{1}{2} \frac{d_{\mathrm{p} 1} d_{\mathrm{p} 2}\left(e^{-\frac{t 2}{\tau}}-e^{-\frac{t 1}{\tau}}\right)}{\left(d_{\mathrm{p} 1}-d_{\mathrm{p} 2}\right)\left(1-e^{-\frac{t 1}{\tau}}\right)\left(1-e^{-\frac{t 2}{\tau}}\right)} \frac{\Delta T}{R_{\mathrm{th}} V_{\mathrm{p} \Sigma}},
$$

given that thermal parameters $R_{\mathrm{th}}$ and $C_{\mathrm{th}}$ are known from a preceding DC calibration measurement as described in the next subsection.

\section{E. DC Calibration}

By means of capturing the temperature response of the sample subject to a stepwise impressed power loss of pre-determined value, the parameters of the model (12) can be identified by means of a least mean square regression. For good predictions of the model, it is crucial that the power range covered in the calibration actually resembles the core loss occurring in the respective operating point of interest, $\{\hat{B}, f\}$. Fig. 12(a) shows the excellent agreement between the measured temperature response obtained for the solid sample (blue) and the simulated response of the mathematical model with identified parameters (red) for several impressed power levels ranging from $0.5 \mathrm{~W}$ to $7 \mathrm{~W}$. Treating the solid ferrite sample as an electric conductor with a DC resistance, $R_{\mathrm{DC}}$, the power loss required for the calibration measurements can be impressed by means of Joule heating, $P_{\mathrm{DC}}=R_{\mathrm{DC}} I_{\mathrm{DC}}^{2}$. The measured DC resistance of the solid sample amounts to $5.5 \mathrm{k} \Omega$ at room temperature and varies strongly with temperature as shown in Fig. 12(b). Since the temperature of the sample rises during the measurement but the impressed power must be kept constant (step response), feedback control is employed to tightly track $P_{\mathrm{DC}}$ reference by means of adjusting the applied DC voltage as schematically depicted in Fig. 12(c). Since DC quantities are involved, the power instrumentation is trivial. Terminals to connect the leads of the DC supply are formed with narrow stripes of $50 \mu \mathrm{m}$ thick copper foil glued to both ends of the sample using conductive silver epoxy. Using thin copper foil for the contacts allows the sample to be properly installed in the magnetic test circuit during the calibration which is crucial to correctly identify the thermal resistance to ambient. The identified parameters, $C_{\mathrm{th}}=3.83 \mathrm{~J} / \mathrm{K}$ and $R_{\mathrm{th}}=37.8 \mathrm{~K} / \mathrm{W}$ are in reasonable agreement with estimates based on material constants as listed in Table II $\left(7 \cdot C_{\mathrm{th}, \mathrm{p}} \approx 3.6 \mathrm{~J} / \mathrm{K}, 1 / 2 \cdot R_{\mathrm{th}, \mathrm{a}}=58.9 \mathrm{~K} / \mathrm{W}\right)$.

\section{F. Measurement Error}

Consolidating (13) and (14), two main sources of error can be identified: (i) The IR camera's standard deviation of the 
temperature difference measurement $\Delta T$ denoted with $\sigma_{\Delta \mathrm{T}}$ and (ii) uncertainty in the parameters $R_{\mathrm{th}}, C_{\mathrm{th}}$ of the underlying model represented with standard deviations $\sigma_{\mathrm{R}}$ and $\sigma_{\mathrm{C}}$. Potential uncertainty in the measured time intervals due to sampling delay and/or jitter of the IR camera is neglected because of the high sampling frame rate relative to the measurement time. In order to calculate the error propagation, the non-linear equations of $\kappa$ and $\kappa_{\mathrm{S}}$ are linearized around $\chi=\left(t_{1 \chi}, t_{2 \chi}, \kappa_{\chi}, \tilde{\kappa}_{\mathrm{s} \chi}\right)$ resulting from a specific excitation $\left\{\hat{B}_{\chi}, f_{\chi}\right\}$. In the following the equations are only explicitly presented for $\kappa$ for the sake of brevity. The standard deviation of $\kappa$ around $\chi$ is then given by [30]

$$
\sigma_{\kappa}(\chi)=\sqrt{J_{\kappa}(\chi) \sum J_{\kappa}(\chi)^{T}}
$$

where $\sum$ denotes the covariance matrix and $J_{\kappa}(\chi)$ is the Jacobimatrix of $\kappa$ at $\chi$,

$$
J_{\kappa}(\chi)=\left[\left.\frac{\partial \kappa}{\partial \Delta T}\right|_{\chi},\left.\frac{\partial \kappa}{\partial R_{\mathrm{th}}}\right|_{\chi},\left.\frac{\partial \kappa}{\partial C_{\mathrm{th}}}\right|_{\chi}\right] .
$$

The covariance matrix is given by

$$
\sum=\left(\begin{array}{ccc}
\sigma_{\Delta \mathrm{T}}^{2} & r_{\Delta \mathrm{T}, \mathrm{R}}^{2} \sigma \Delta \mathrm{T} \sigma \mathrm{R} & r_{\Delta \mathrm{T}, C}^{2} \sigma \Delta \mathrm{T} \sigma \mathrm{C} \\
r_{\Delta \mathrm{T}, \mathrm{R}}^{2}{ }_{\Delta \mathrm{T}} \sigma \mathrm{R} & \sigma_{\mathrm{R}}^{2} & r_{\mathrm{R}, \mathrm{C}}^{2} \sigma_{\mathrm{R}} \sigma \mathrm{C} \\
r_{\Delta \mathrm{T}, \mathrm{C}}^{2} \sigma \Delta \mathrm{T} \sigma \mathrm{C} & r_{\mathrm{R}, \mathrm{C}}^{2} \sigma_{\mathrm{R}} \sigma \mathrm{C} & \sigma_{\mathrm{C}}^{2}
\end{array}\right),
$$

where $r_{\mathrm{x}, \mathrm{y}}$ represents the correlation coefficient between stochastic variables $x$ and $y$. From a physical standpoint, there is only a weak correlation between variations in $R_{\mathrm{th}}$ and $C_{\mathrm{th}}$ and also between variations in $\Delta T$ and $R_{\mathrm{th}}$. However, there is a more pronounced negative correlation between variance in $\Delta T$ and $C_{\mathrm{th}}$, since a larger $\Delta T$ during calibration with a specific impressed power and measurement time interval leads to smaller $C_{\mathrm{th}}$. Instead of presenting exhaustive analytical equations, some general facts should be highlighted. Naturally, a distinct difference in plate thickness between the examined multi-gap samples results in a significant difference in elapsed time to reach $\Delta T$ and thus supports a small measurement error.

Since the measurement time $t_{1 \chi}$ and $t_{2 \chi}$ for a specific $\Delta T$ increases inversely proportional to the dissipated power in the ferrite plate, a small (large) absolute error must be expected at a low (high) loss operating point, respectively. In principle, allowing a larger temperature difference $\Delta T$ increases the measurement time and leads to a reduction in error. However, $\Delta T$ cannot be increased too much (here $\Delta T=2.5^{\circ} \mathrm{C} \mathrm{cf}$., Section III-B) since the ferrite properties are a strong function of temperature. In order to calculate a worst case bound on the measurement error a deviation of $\sigma_{\Delta \mathrm{T}}=0.3{ }^{\circ} \mathrm{C}$ and a $15 \%$ deviation of the thermal parameters from their expected values as listed in Table II is assumed. Moreover, since in (17) the exact correlation between the variables is unknown, a worst case error of $\kappa$ and $\tilde{\kappa}_{\mathrm{s}}$ is determined by varying the coefficient $r_{\mathrm{x}, \mathrm{y}}$ in a numerical study. It then follows from (15) that for the operating points of interest, the worst case rel. measurement

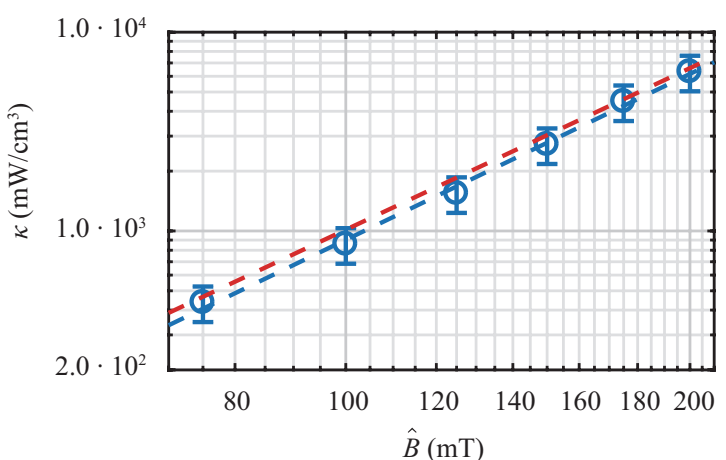

(a)

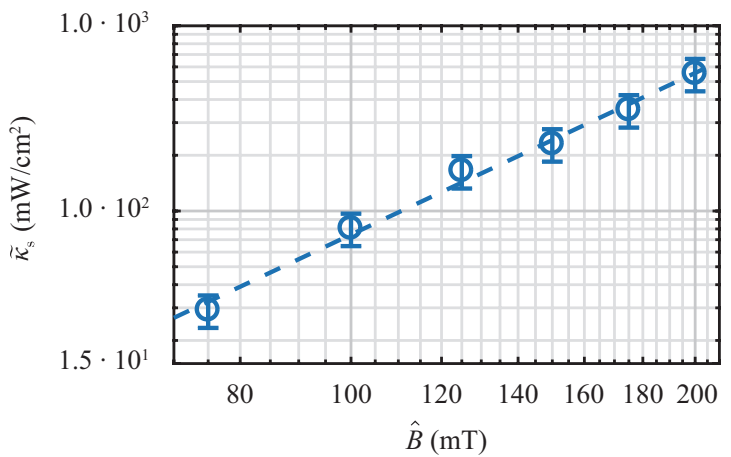

(b)

Fig. 13. Experimentally determined (a) bulk and (b) surface core loss density of MnZn ferrite material 3F4 at a fixed frequency of $400 \mathrm{kHz}$ and varying flux density amplitude. The prediction obtained from the Steinmetz model fitted to the experimental data is indicated by the dashed blue line. In addition the prediction with Steinmetz parameters provided by the vendor (cf., Table III, [31]) is indicated by the dashed red line in (a).

error is estimated to be

$$
\frac{\sigma_{\kappa}}{\kappa}(\chi) \leqslant 20.3 \% \text { and } \frac{\sigma_{\tilde{\kappa} \mathrm{s}}}{\tilde{\kappa}_{\mathrm{s}}}(\chi) \leqslant 19.9 \% \text {. }
$$

\section{EXPERIMENTAL RESULTS}

In this section the bulk and surface core loss density of $\mathrm{MnZn}$ ferrite material 3F4 of Ferroxcube are presented. The experimental results were obtained by means of the thermometric measurement setup with IR camera as described in detail in Section III-B and expression (13) and (14) based on the exponential thermal model. The measurements were carried out for a sinusoidal flux density with amplitudes varying from $75 \mathrm{mT}$ up to $200 \mathrm{mT}$ and excitation frequencies ranging from $200 \mathrm{kHz}$ up to $1 \mathrm{MHz}$. For this range of operating points $\{\hat{B}, f\}$, the worst case relative measurement error of both bulk and surface core loss density is limited to $20 \%$ as discussed in the previous section.

The bulk and surface core loss density at a fixed frequency of $400 \mathrm{kHz}$ and varying flux density amplitudes is depicted in Fig. 13(a) and (b). The predictions obtained from the Steinmetz model (1) fitted to the experimental data and from parameters provided by the vendor are represented by the dashed blue and red line, respectively. The experimental and vendor parameter 
TABLE III

STeinMetZ PARAMETERS For

MnZn Ferrite Material 3F4 From Ferroxcube $[\kappa]=\mathrm{mW} / \mathrm{cm}^{3}$ and $\left[\kappa_{\mathrm{s}}\right]=\mathrm{mW} / \mathrm{cm}^{2}$

\begin{tabular}{lccc}
\hline \hline & $k$ & $\alpha$ & $\beta$ \\
\hline$\kappa$ & $1.32 \times 10^{-2}$ & 1.36 & 2.77 \\
$\kappa^{\mathrm{a}}$ & $35 \times 10^{-2}$ & 1.1 & 2.7 \\
$\kappa^{\mathrm{b}}$ & $1.2 \times 10^{-4}$ & 1.7 & 2.7 \\
$\kappa_{\mathrm{s}}$ & $2.72 \times 10^{-2}$ & 1.13 & 2.9 \\
\hline \hline
\end{tabular}

${ }^{\mathrm{a}}[31], 100 \mathrm{kHz}-600 \mathrm{kHz}$ and $30^{\circ} \mathrm{C}$

[31],600 kHz-1000 kHz and $30^{\circ} \mathrm{C}$

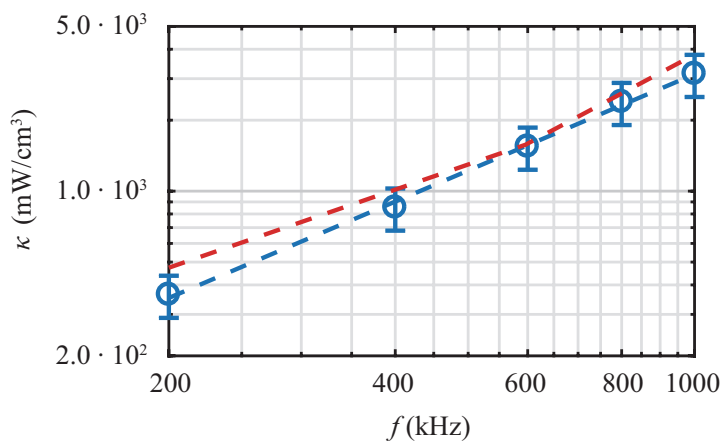

(a)

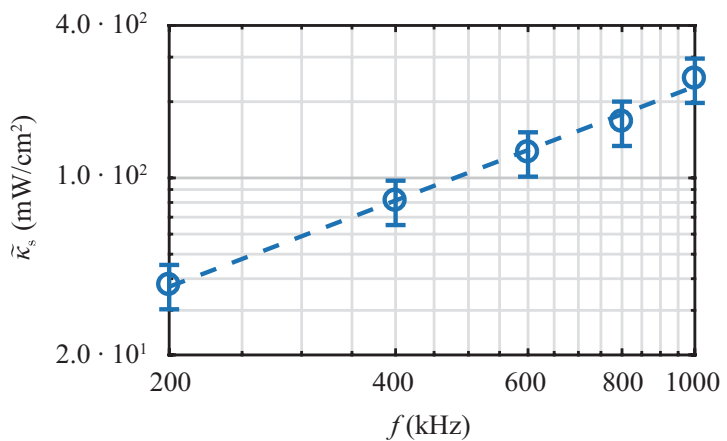

(b)

Fig. 14. Experimentally determined (a) bulk and (b) surface core loss density of $\mathrm{MnZn}$ ferrite material 3F4 at a fixed peak flux density of $100 \mathrm{mT}$ and varying frequency. The prediction obtained from the Steinmetz model fitted to the experimental data is indicated by the dashed blue line. In addition the prediction with Steinmetz parameters provided by the vendor (cf., Table III, [31]) is indicated by the dashed red line in (a).

of the Steinmetz model are summarized in Table III. In a similar manner, the bulk and surface core loss density at a fixed flux density amplitude of $100 \mathrm{mT}$ and varying excitation frequency is depicted in Fig. 14(a) and (b). Note that depending on the frequency range a different set of vendor Steinmetz parameter is employed which explains the slight kink in the dashed red line at $600 \mathrm{kHz}$ as indicated in Fig. 14(a). However, the Steinmetz fit to the experimental data was performed over the entire frequency range which explains the discrepancy, especially in the frequency exponent $\alpha$, between experimental and vendor parameters (cf., Table III). It can be seen from Fig. 13(b) and Fig. 14(b) that the measured surface loss density varies from $30 \mathrm{~mW} / \mathrm{cm}^{2}$ to $600 \mathrm{~mW} / \mathrm{cm}^{2}$ for the considered operating points.

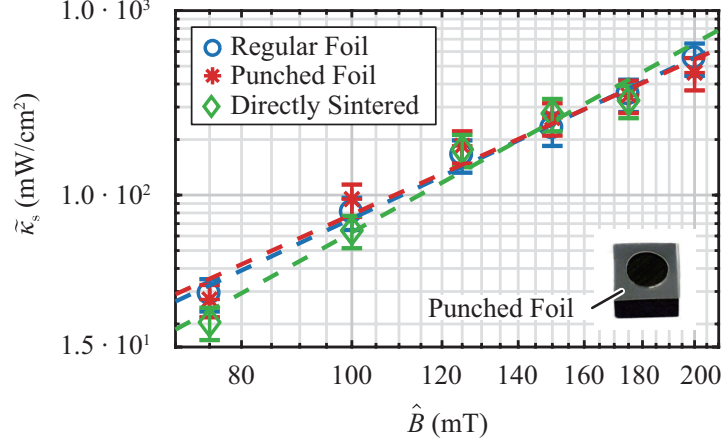

Fig. 15. Comparison of the surface loss density of different multi-gap samples at a fixed frequency of $400 \mathrm{kHz}$ and varying flux density amplitude. The regular sample is composed of $7 \times 3 \mathrm{~mm}$ plates with $100 \mu \mathrm{m}$ Mylar foil in between. Roughly half of the Mylar foil area was removed in the case of the punched foil sample. The green grinded sample was constructed from ferrite plates which were pressed and then sintered to the desired dimensions without machining.

In order to assess whether the Mylar foil inserted between the plates has any contribution to the measured surface loss (e.g., by magnetostriction introduced friction), a multi-gap sample was prepared using Mylar foil with a punched round hole of approx. $5 \mathrm{~mm}$ diameter in the center of each foil as illustrated in the bottom right of Fig. 15. Punching a hole in the center ensures the desired gap distance and therefore still ensures equal flux distribution and/or prevents partial saturation while removing roughly $50 \%$ of the gap material. The measured surface loss density of a multi-gap sample with punched Mylar foil is compared to the regular multi-gap sample in Fig. 15, showing no difference between the results. To understand whether or not machining is actually the main cause of the increased core loss, ferrite plates directly sintered to the final dimension were provided by Ferroxcube in this study. These plates were manufactured by pressing and then machining ferrite powder into the appropriate dimensions before the actual sintering was performed (green grinding). Taking the size shrinkage caused by the sinter process into account, the correct final dimension of the plates has been immediately achieved eliminating the need of an additional machining step. The experimental measured surface loss density is also included in Fig. 15. It can be seen that $\tilde{\kappa}_{\mathrm{s}}$ has slightly reduced at low flux density amplitudes in comparison to the regular sample composed of machined ferrite plates and exhibits a steeper slope corresponding to a Steinmetz parameter of $\beta_{\mathrm{s}}=3.4$.

\section{DisCUSSION}

The measured core loss densities shown in Fig. 13(a) and Fig. 14(a) show good agreement with the Steinmetz model provided by the manufacturer validating the proposed calorimetric core loss measurement technique. With regard to the surface loss densities (cf., Fig. 13(b) and Fig. 14(b)), the experimental data can be also well predicted with the Steinmetz model. Interestingly and as to be expected, the Steinmetz parameters of the surface layer are slightly different from the bulk material as listed in Table III. Compared to the bulk ferrite, the frequency exponent $\alpha$ in the surface is smaller $(1.36 \rightarrow 1.13)$ 


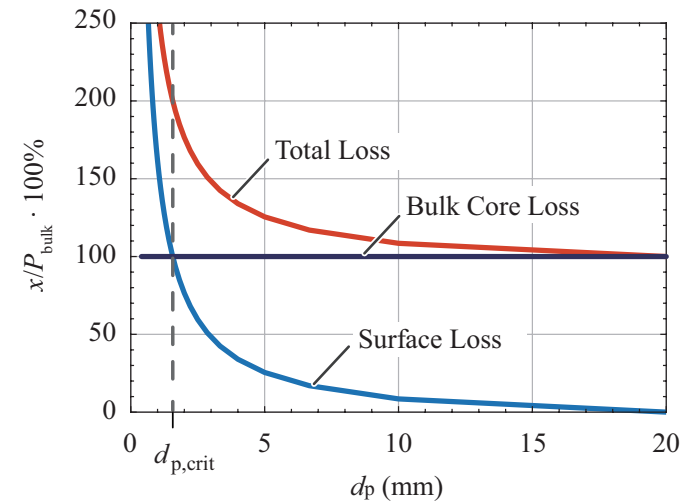

(a)

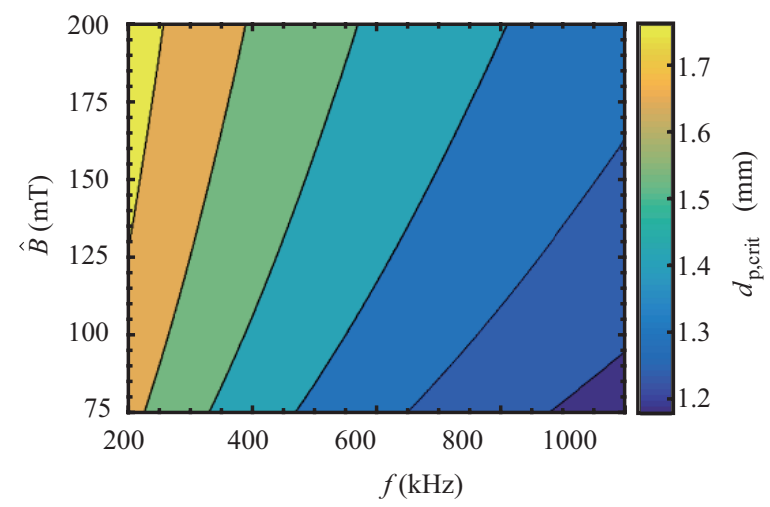

(b)

Fig. 16. (a) Surface and total core loss of a composite core assembled with individual $d_{\mathrm{p}}$ thick plates relative to the bulk core loss of a solid ferrite piece with $d_{\text {bulk }}=20 \mathrm{~mm}$ total length based on the experimental results obtained for $3 \mathrm{~F} 4$ at $125 \mathrm{mT}$ and $400 \mathrm{kHz}$. The critical plate thickness $d_{\mathrm{p}, \mathrm{crit}}$ is defined to be reached when the surface loss is equal to the bulk core loss. (b) Critical thickness of the examined $3 \mathrm{~F} 4$ material depending on the actual operating point $\{\hat{B}, f\}$

and the flux density exponent $\beta$ is larger $(2.77 \rightarrow 2.9)$. Since the loss in the deteriorated surface layer is more sensitive to variation in flux density than it is to variation in frequency (almost linear relationship with frequency), it can be argued that hysteresis losses are more pronounced in the surface layer.

In order to illustrate the impact of $\widetilde{\kappa}_{\mathrm{s}}$ in more practical terms, the total power loss of a composite core assembled from individual $d_{\mathrm{p}}$ thick plates relative to the bulk core loss of a solid ferrite piece with $d_{\text {bulk }}$ total length is computed,

$$
\begin{aligned}
\frac{P_{\text {tot }}}{P_{\text {bulk }}} & \approx \frac{\left(\kappa d_{\text {bulk }} A_{\mathrm{p}}+\kappa_{\mathrm{s}} 2 d_{\mathrm{s}} \frac{d_{\text {bulk }}}{d_{\mathrm{p}}} A_{\mathrm{p}}\right)}{\kappa d_{\text {bulk }} A_{\mathrm{p}}} \\
& =1+\frac{\kappa_{\mathrm{s}}}{\kappa} \frac{2 d_{\mathrm{s}}}{d_{\mathrm{p}}}=1+\frac{\tilde{\kappa}_{\mathrm{s}}}{\kappa} \frac{2}{d_{\mathrm{p}}},
\end{aligned}
$$

and depicted in Fig. 16(a) based on the experimental results obtained for 3F4 at $125 \mathrm{mT}$ and $400 \mathrm{kHz}$. The reference sample is constructed from a single piece of ferrite with a total length of $20 \mathrm{~mm}$ exhibiting only bulk core loss. With decreasing height of the ferrite plate, more and more plates must be stacked to reach the same length as the solid reference sample. Consequently, the total losses increase since more and more deteriorated surface layers are introduced in the sample. Note that the ratio between total and bulk core loss given by (19) is a function of only the plate thickness, $d_{\mathrm{p}}$, since both bulk and surface core losses increase proportional with the core cross section, $A_{\mathrm{p}}$, and the total sample length, $d_{\text {bulk }}$, assuming that $d_{\text {bulk }}$ is varied in multiples of $d_{\mathrm{p}}$. To further clarify, two composite core samples with different cross section area and/or different total sample length will exhibit the same total to bulk loss ratio (19) if the samples are constructed of plates with identical thickness. The critical plate thickness $d_{\text {p,crit }}$ is defined to be reached when the surface loss is equal to the bulk core loss, that is to say when the total power loss has doubled compared to the solid (singlepiece) sample. In the case at hand, a composite core with 13 stacked $1.5 \mathrm{~mm}$ plates will exhibit roughly twice the core loss compared to the single-piece reference sample. Since $\kappa$ and $\tilde{\kappa}_{\mathrm{s}}$ exhibit a different dependency on flux density amplitude and frequency (cf., Table III), the critical plate thickness varies depending on the actual operating point $\{\hat{B}, f\}$ as depicted in Fig. 16(b). It should be noted that, the actual critical thickness depends not only on the properties of the ferrite but also on the exerted mech. stress during the machining of the plates. In an open discussion of the findings with the scientific community prior to this publication, it was argued that the measured excess core loss in the multi-gap core is actually the result of microvibrations causing friction between plate surface and gap material and that these micro-vibrations are excited by means of magnetostriction as discussed in detail in [32]-[34]. In this respect, if friction between plate surfaces and Mylar would indeed be the reason for the measured surplus core losses, then in case of the sample using punched Mylar foil, where roughly half of the interaction area was removed, a significant reduction in $\widetilde{\kappa}_{\mathrm{s}}$ should be observable. However, as depicted in Fig. 15, the measured surface loss density of the multi-gap sample with punched and regular Mylar foil is essentially identical, which strongly contradicts the notion that micro-vibration are the origin of the excess core losses. Furthermore, an experimental assessment with a laser vibrometer (Polytec CLV-2534) confirmed that within the considered excitation frequency range no micro-vibrations are excited.

Moreover, the idea was put forward that the measured excess core loss might be associated with machining and assembly tolerances. For instance, a pronounced tilt between the plates of the multi-gap assembly causes a non-uniform gap length which has a strong impact on the core loss due to flux crowding, especially if the assembly is composed of very thin plates and/or features small gap lengths. However, as analyzed in Appendix A, for a worst case machining and assembly tolerance, the plate thickness and gap length can be chosen such to minimize the resulting impact of flux crowding. The combination of using $3 \mathrm{~mm}$ plates and $100 \mu \mathrm{m}$ gap length to implement the multigap sample examined in this study, results in a negligible contribution of flux crowding to the measured excess core loss in the multi-gap sample.

Very intriguing is also the fact that directly sintered plates still exhibit excess core loss in the same order of magnitude as the 
machined plates which contradicts the hypothesis that mech. stress during machining is introducing the excess loss. However, it can be argued that the directly sintered plates also feature a layer of deteriorated ferrite performance close to the surfaces. These deteriorated layers are not caused by mech. stress introduced during machining but from an altered $\mathrm{Fe}^{2+} / \mathrm{Fe}^{3+}$ ion concentration in the iron oxide close to the surface. Among other factors, the oxygen concentration in the atmosphere during sintering strongly affects the balance between $\mathrm{Fe}^{2+}$ and $\mathrm{Fe}^{3+}$ ions. Since the plate surfaces are directly exposed to the atmosphere inside the controlled temperature ferrite processing chamber in contrast to the bulk, and a deviation from the ideal, temperature dependent $\mathrm{O}_{2}$ concentration is inevitable in a practical setting. Thus, a shallow ferrite layer close to the surface is likely to exhibit adverse magnetic properties which is an explanation why there is also excess core loss present in the case of the directly sintered plates.

\section{CONCLUSION}

A multi-gap inductor design can potentially reduce winding losses due to a reduction in the magnetic air gap leakage field. Unfortunately, the manufacturing of a multi-gap core composed of multiple stacked MnZn ferrite plates - can lead to an increase of core loss which potentially outweighs the saving in winding conduction loss. Based on the literature, the dominating cause of the excess core loss is shallow layers of deteriorated magnetic performance just underneath the plate surfaces. A sophisticated calorimetric measurement approach based on temperature rise monitoring was developed, allowing to differentiate between and quantify bulk and surface core loss densities. Experimental results were presented for the MnZn ferrite material 3F4 from Ferroxcube. Reasons for the deteriorated surface are mechanical stress exerted during machining of the plates as suggested by the literature and nonideal conditions during sintering as suggested by the vendor. Several post machining treatments proposed in the literature annealing, etching and stress-free polishing with colloidal silica - have been applied to the machined plates in order to restore the original ferrite properties and reduce core loss, unfortunately without significant success so far. Accordingly, more work needs to be done, especially considering an annealing treatment with defined atmosphere and temperature profile recommended by the vendor. Moreover, NiZn ferrite should be examined in future work since it is known from the literature that low- $\mu$ materials are less sensitive to mechanical stress. Thus, a multigap design using NiZn ferrite might not suffer from increased core losses as also the recent work in [35] seems to indicate.

\section{APPENDIX A}

\section{Influence of Assembly and Machining Tolerances}

In the multi-gap inductor design described in the introduction of this paper, the center limb of the E-type core is composed of thin ferrite plates stacked on top of each other. These plates are either cut from a long ferrite bar by means of a diamond saw or are manufactured by pressing ferrite powder into the desired shape prior to sintering. Due to mechanical tolerance in the manufacturing processes, it is possible that the plate surfaces exhibit a convex or concave curvature or are not ideally coplanar. In order to establish the correct gap length, a non-magnetic spacer material is inserted between the plates. The spacer, also intended to glue adjacent plates together, can be realized by means of a plastic film (e.g., Kapton or Mylar) with double sided adhesive or by a mix of epoxy resin with spherical silica powder as outlined in Appendix B, where the latter allows gap lengths below $30 \mu \mathrm{m}$. Due to mechanical tolerances, it is possible that the plates in the stack are not ideally coplanar but slightly tilted. In order to estimate how much these machining and assembly tolerances affect the core losses, a 2D FEM study was conducted. The static flux density distribution within the stacked plates considering a linear core material $\left(\sigma=0, \epsilon_{\mathrm{r}}=1, \mu_{\mathrm{r}}=3000\right)$ is computed based on Ampères's Law. In Fig. 17(a), the impact of several machining/assembly tolerances on the flux density distribution is shown. The ideal multi-gap core, composed of $15 \times 1 \mathrm{~mm}$ thick plates with a 30 $\mu \mathrm{m}$ gap, is depicted at the very left indicating a homogeneous flux density distribution. The flux density component normal to the plate surface (y-direction) averaged with respect to the plate width (x-direction) in the very center of the stack (cf., red line in the image on the very left of Fig. 17(a)) for one ampereturn of excitation is chosen as benchmark for an insightful comparison in per unit values. Since the effective permeability of the composite core changes depending on the magnitude of the simulated non-ideality, the excitation current is adopted accordingly such that the avg. flux density in y-direction in the center of the stack is identical under all considered scenarios. Introducing variable $d_{\Delta}$ to model tilt, curvature and noncoplanarity arising from mech. tolerances, leads to a pronounced flux crowding as it can be seen from the FEM simulation results in Fig. 17(a). The impact of flux crowding on the actual core loss is estimated considering $p \propto B_{\mathrm{pu}}^{\beta}$ according to (1), where $\beta=2.4$ in this study. The avg. loss density of the multi-gap core is then given by,

$$
\bar{p}=\frac{1}{A} \cdot \oiint_{A} p \mathrm{~d} A=\frac{1}{A} \cdot \oiint_{A} B^{\beta} \mathrm{d} A
$$

where $A$ denotes the total core area of the stack. Fig. 17(b) shows the avg. loss density for a fixed plate thickness and gap length $\left(d_{\mathrm{p}}=1 \mathrm{~mm}, d_{\text {gap }}=30 \mu \mathrm{m}\right)$ but for a varying deviation. A tilt between plates has the most significant impact and results in an increase of the avg. loss density up to a factor of 2.3 for the worst considered error of $d_{\Delta}=50 \mu \mathrm{m}$. Note that the effective difference in gap length measured at both ends of the plate is actually twice $d_{\Delta}$ as indicated in Fig. 17(a). Fig. 17(c) depicts the avg. core loss density as a function of the gap length $d_{\text {gap }}$ for a fixed plate thickness and the worst case error. It becomes evident that increasing the individual gap length allows to mitigate the impact of the error on the loss density. Likewise, Fig. 17(d) depicts how the loss density reduces when the stack is composed of thicker plates. It can be concluded from this study that even if all plates feature undisturbed ferrite properties, an excess of core loss can still be observed in a multi-gap inductor due to machining and assembly tolerances. A deviation of $d_{\Delta}=50 \mu \mathrm{m}$ might reflect a worst case scenario, but it is reasonable to expect a deviation 

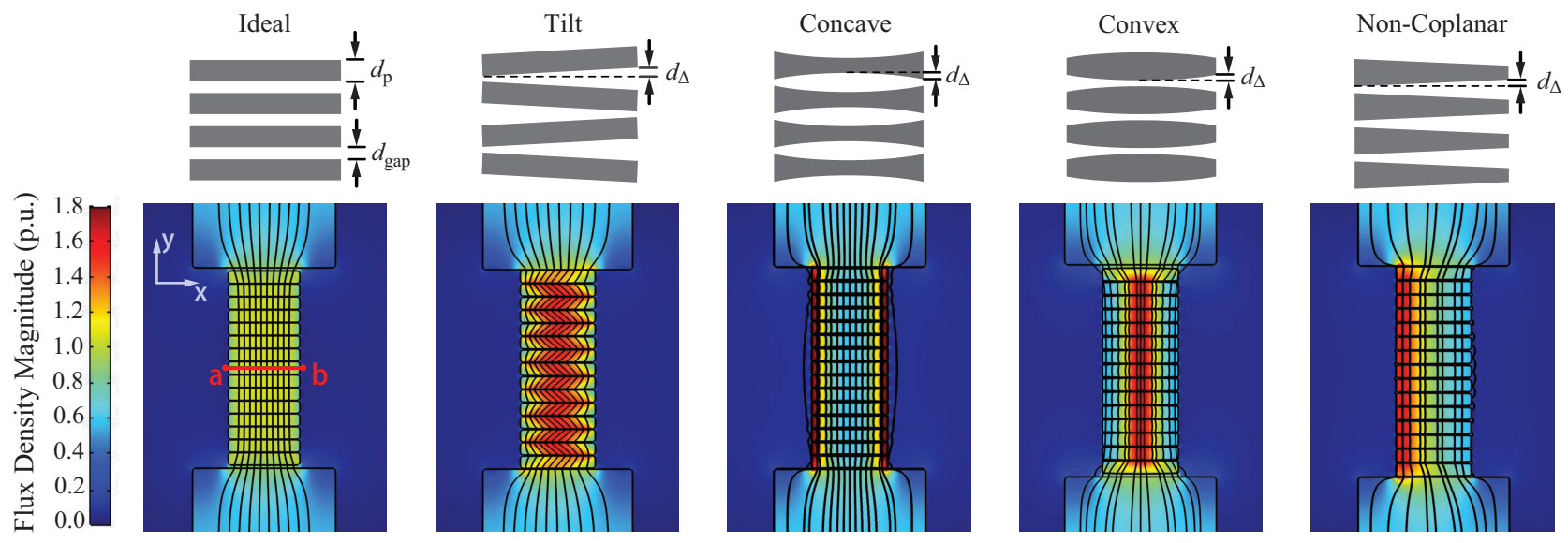

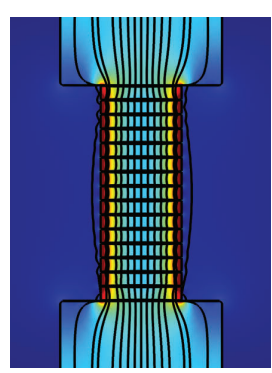

$d_{\Delta}=50 \mu \mathrm{m}$

(a)

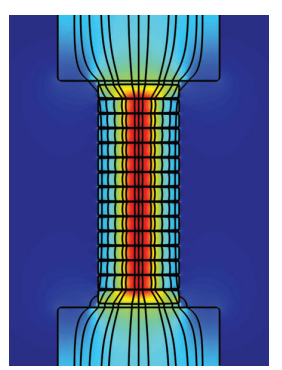

$d_{\Delta}=50 \mu \mathrm{m}$
$d_{\Delta}=50 \mu \mathrm{m}$

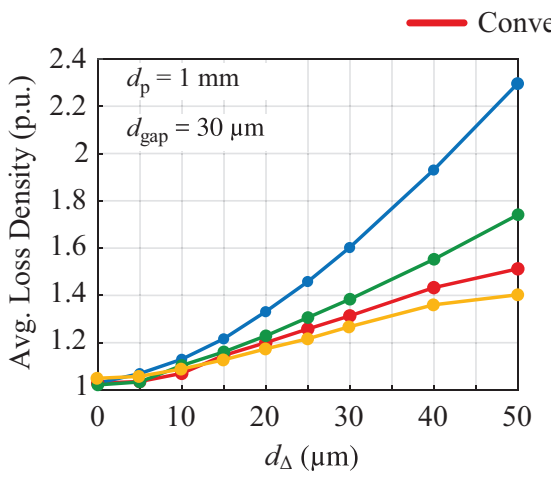

(b)

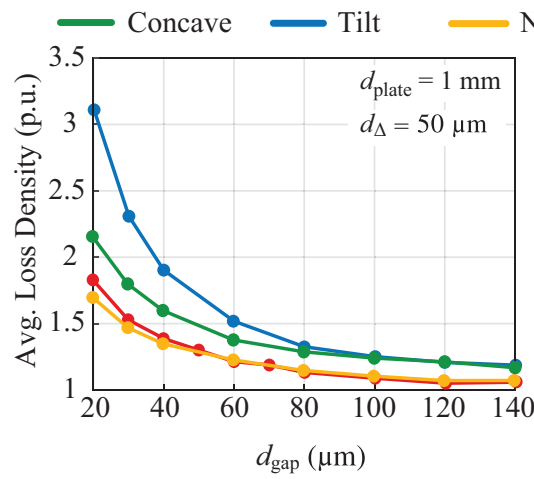

(c)

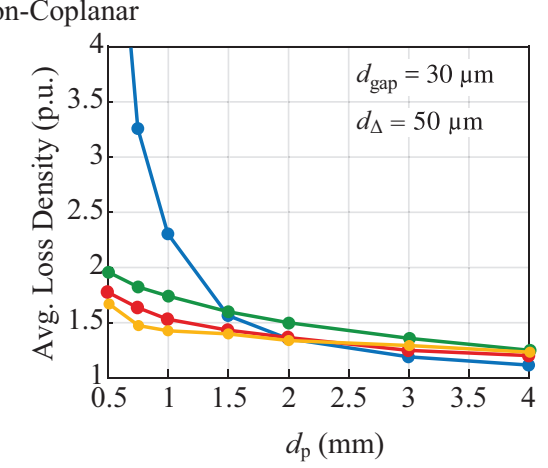

(d)

Fig. 17. (a) 2D FEM field simulation results indicating the impact of several machining / assembly tolerances on the flux density distribution in the sample. The ideal multi-gap core shown on the left side is composed of $15 \times 1 \mathrm{~mm}$ thick plates with a $30 \mu \mathrm{m}$ gap and serves as reference for the p.u. calculation. (b) Avg. core loss density in the sample for a fixed plate thickness and gap length $\left(d_{\mathrm{p}}=1 \mathrm{~mm}, d_{\text {gap }}=30 \mu \mathrm{m}\right)$ but for a varying deviation $d_{\Delta}$. (c) Avg. core loss density in the sample as a function of the gap length $d_{\text {gap }}$ for a fixed plate thickness $d_{\mathrm{p}}$ and the worst case error $\left(d_{\Delta}=50 \mu \mathrm{m}\right)$. (d) Avg. core loss density in the sample as a function of the plate thickness $d_{\mathrm{p}}$ for a fixed gap length $d_{\text {gap }}$ and the worst case error $\left(d_{\Delta}=50 \mu \mathrm{m}\right)$.

of $d_{\Delta}=10 \mu \mathrm{m}-20 \mu \mathrm{m}$ in practice. For instance the standard deviation in plate thickness measured with a precision caliper at all four lateral faces amounts to $5 \mu \mathrm{m}$ in the case of the machined plates and to $23.7 \mu \mathrm{m}$ in the case of the direct sintered plates (cf., Section IV and Section V) provided by Ferroxcube for this study. In order to isolate and correctly quantify surface related core loss, the multi-gap samples employed to obtain the experimental data presented in Section IV of this paper are constructed with $3 \mathrm{~mm}$ thick plates and $100 \mu \mathrm{m}$ Mylar foil which limits the impact of mech. tolerances on the measured core loss to less than $5 \%$ for the (unlikely) worst case error.

\section{ApPendix B}

\section{Core Loss Increase Caused by Pressure Buildup}

To ensure mechanical stability of the multi-gap inductor, the ferrite plates constituting the center limb have to be adhered to each other. The two-component adhesive Duralco 4460, which is based on epoxy resin was chosen for this task. Before application, the adhesive was mixed with spherical silica powder to provide a defined gap length [3]. Although the volume shrinkage of the glue is only $0.5 \%$ [36], the ferrite material is exposed to mechanical stress after this manufacturing process. Furthermore, since the cured glue shows a very low viscosity, the difference in thermal expansion coefficients of the glue (CTE of $64 \mathrm{ppm} / \mathrm{K}$ [36]) and the ferrite (CTE is approximately $12 \mathrm{ppm} / \mathrm{K}$ ) can cause additional mechanical stress on the ferrite material. The impact of mechanical compression on the permeability and the saturation flux density was investigated in several publications, e.g., [37]-[39]. However, these property changes are not critical in the case of the designed multigap inductor (cf., Table I), since the effective permeability of the magnetic circuit is mainly determined by the length of the gap. Here, the most important observed parameter shift due to exerted mechanical stress on the ferrite is the increase in hysteresis losses. In [40] this effect was investigated on molded ferrites. E cores were glued on a PCB substrate and encapsulated using a transfer mold process at a temperature of $175^{\circ} \mathrm{C}$ and a process pressure of approximately $10 \mathrm{MPa}$. The core losses before and after this technological process were determined electrically by applying the measurement principle described in [41] and reviewed in Appendix D of this paper. It was observed that the core losses of the investigated ferrite materials are increased after the molding process. Fig. 18(a) 


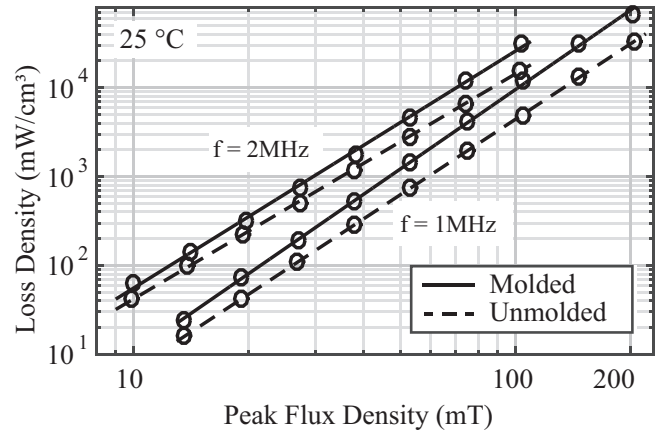

(a)

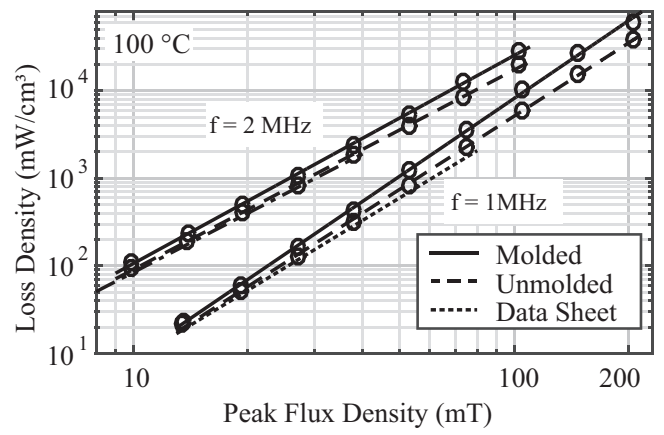

(b)

Fig. 18. Impact of molding on the core loss density of $\mathrm{MnZn}$ ferrite material $3 \mathrm{~F} 4$ at (a) $25^{\circ} \mathrm{C}$ and (b) at $100^{\circ} \mathrm{C}$.

and (b) depict the measured results for $3 \mathrm{~F} 4$ at $25^{\circ} \mathrm{C}$ and $100{ }^{\circ} \mathrm{C}$, respectively (in [40] material $\mathrm{G}$ was examined). The results show a more distinctive increase of core loss at higher flux densities, e.g., increase of core loss after molding at $1 \mathrm{MHz}$, $100{ }^{\circ} \mathrm{C}$ and $20 \mathrm{mT}$ by a factor of 1.2 and at $1 \mathrm{MHz}, 100{ }^{\circ} \mathrm{C}$ and $150 \mathrm{mT}$ by a factor of 1.7. Due to the larger share of eddy current losses at higher frequencies, the dependency of the core loss increase due to mechanical compression on the frequency is lower, e.g., an increase of core loss after molding at $100 \mathrm{mT}$, $100{ }^{\circ} \mathrm{C}$ and $1 \mathrm{MHz}$ by a factor of 1.74 and at $100 \mathrm{mT}, 100{ }^{\circ} \mathrm{C}$ and $2 \mathrm{MHz}$ by a factor of only 1.45 occurs. It is concluded in [40] that only the hysteresis losses are increased by mechanical stress on the material. Furthermore, it is worth noting that the increase of the core losses is lower at a temperature of $100^{\circ} \mathrm{C}$ compared to $25^{\circ} \mathrm{C}$. How much the additional hysteresis losses in the stack of ferrite plates can be reduced by using a more elastic adhesive, for instance Duralco 4538 with a shore hardness of only D70 [36], is the subject of ongoing research.

\section{APPENDIX C}

\section{Ohmic Conduction Loss in Ferrite}

Another origin of core loss in the multi-gap inductor design discussed in Section I is related to the finite conductivity of the $\mathrm{MnZn}$ ferrite and the high parasitic capacitance between the winding packages (cf., Fig. 1(a) and (b)) and the outer magnetic core. The effect can be explained by means of Fig. 19(a).

Between both outer turns of the top and bottom winding package and the ferrite of the magnetic return path (outer limb) a high parasitic capacitance is formed due to the comparably

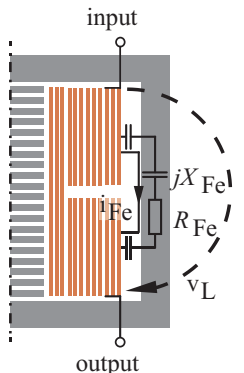

(a)

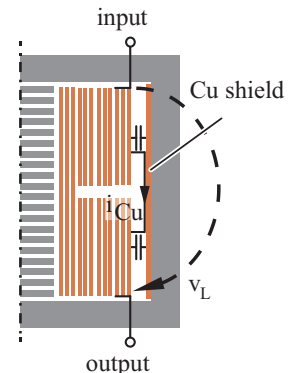

(b)

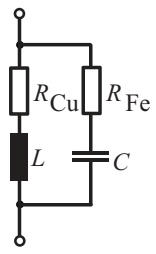

(c)
Fig. 19. (a) Explanation of the conduction loss effect in the ferrite material and (b) measure to minimize these additional losses by employing a low impedance copper shield. (c) Equivalent circuit of the inductor with winding resistance $\left(R_{\mathrm{C}}\right.$ and $L)$ and parasitic conduction path connected in parallel $\left(R_{\mathrm{Fe}}\right.$ and $\left.C\right)$.

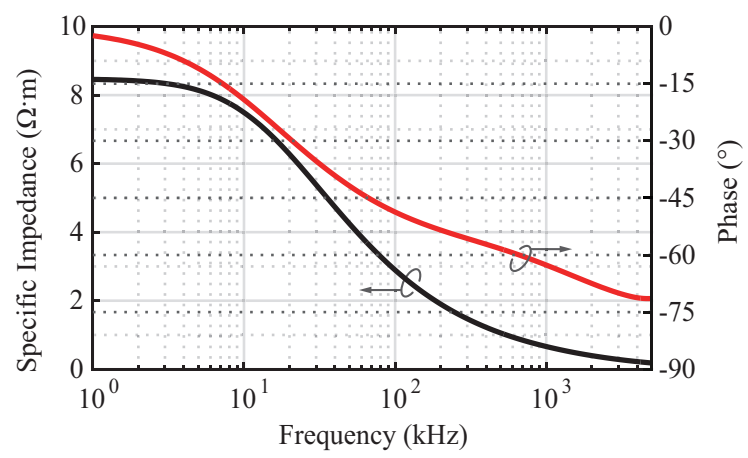

(a)

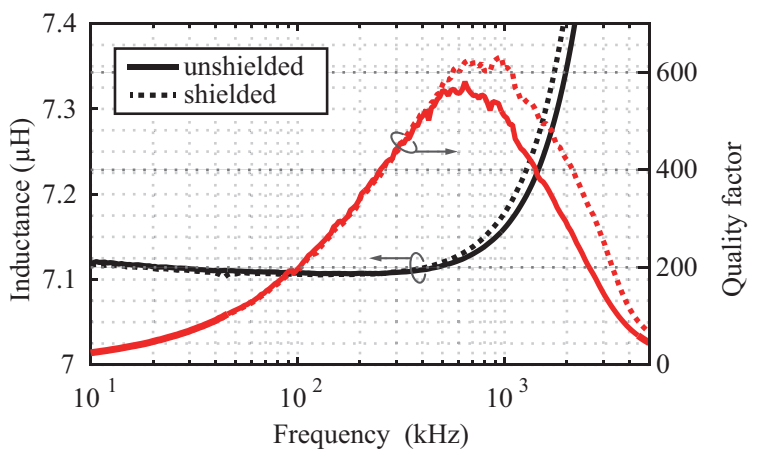

(b)

Fig. 20. (a) Measured specific impedance of the MnZn ferrite material DMR51 and (b) measured inductance and quality factor of the unshielded and shielded inductor.

large surface of the foil winding. By means of a simple plate capacitor model, the capacitance per winding package is estimated to be approximately $120 \mathrm{pF}$ (relative permittivity of the insulation foil: 3.5 , the foil thickness: $60 \mu \mathrm{m}$, surface of one winding package that is covered by ferrite: $240 \mathrm{~mm}^{2}$ ). The voltage occurring between both turns is the total inductor voltage, because the outer turn of the top winding package is the first and the outer turn of the bottom winding package is the last turn of the inductor winding. Therefore, a high parasitic current through the ferrite material occurs at high frequencies and causes additional losses in the ferrite material due to the finite ohmic resistance of the ferrite. Fig. 20(a) shows the measured specific impedance of the MnZn ferrite DMR51. The measurement was performed on a ferrite cuboid (length: 
$8.2 \mathrm{~mm}$, cross section: $\left.(2.6 \times 3.0) \mathrm{mm}^{2}\right)$ using the impedance analyzer Agilent 4294A. The MnZn ferrite material is comprised of grains with a relatively low specific ohmic resistance and layers of high resistivity between these ferrite grains. At frequencies up to $4 \mathrm{kHz}$ the specific impedance is almost exclusively determined by the specific ohmic resistance of the grain boundaries and is approximately $8.3 \Omega \mathrm{m}$. At higher frequencies an additional capacitive current flows across the isolating layers (grain boundaries), thus the impedance features a capacitive imaginary part and the magnitude reduces. The specific impedance of the DMR51 material at a frequency of $1 \mathrm{MHz}$ is only $(0.3-0.58 \mathrm{i}) \Omega \mathrm{m}$.

Considering the geometry of the inductor, the resulting impedance of the average conduction path through the ferrite at a frequency of $1 \mathrm{MHz}$ caused by the described parasitic capacitance is $Z_{\mathrm{Fe}}=R_{\mathrm{Fe}}+j X_{\mathrm{Fe}}=(47-91 i) \Omega$ (length of the conduction path: $9 \mathrm{~mm}$, cross section: $\left.(1.9 \times 30) \mathrm{mm}^{2}\right)$. It follows then that the impedance of the equivalent circuit (cf., Fig. 19(c)) is given by

$$
\begin{aligned}
Z_{\mathrm{eq}} & =\left(R_{\mathrm{Cu}}+j \omega L\right) \|\left(R_{\mathrm{Fe}}+\frac{1}{j \omega C}\right) \\
& \approx(j \omega L) \|\left(R_{\mathrm{Fe}}+\frac{1}{j \omega C}\right),
\end{aligned}
$$

wherein the approximation holds since $\omega L \gg R_{\mathrm{Cu}}$ and parameter $C$ represents the series connection of both parasitic capacitances between the outermost turns of the respective winding package and the outside magnetic core as well as the capacitive part of $Z_{\mathrm{Fe}}(120 \mathrm{pF}\|120 \mathrm{pF}\| 1.7 \mathrm{nF}$ in total $C=58 \mathrm{pF})$. Computing the real part of (21) allows to estimate the impact on the measured ohmic resistance $\Delta R$ of the inductor at a frequency of $1 \mathrm{MHz}$ given by

$$
\Delta R=\Re\left(Z_{\mathrm{eq}}\right)=\frac{R_{\mathrm{Fe}} \cdot(2 \pi f L)^{2}}{R_{\mathrm{Fe}}^{2}+\left(2 \pi f L-\frac{1}{2 \pi f C}\right)^{2}} \approx 14 \mathrm{~m} \Omega .
$$

This is equivalent to an increase of the winding resistance and a decrease of the quality factor by roughly $20 \%$, respectively. Considering a triangular current mode (TCM) inverter designed to comply with the Google Little Box Challenge specifications [7], similar to the converter presented in [6] but with lower output inductance value $(7 \mu \mathrm{H}$ instead of $10 \mu \mathrm{H})$, it is estimated that the ferrite conduction losses amount on average to an additional $240 \mathrm{~mW}$ core loss per inductor. To minimize these additional losses the distance between the outer magnetic core and the winding packages can be increased. However, this leads to an increase in inductor size. A further possibility is to insert a low impedance shielding layer between the ferrite and the winding packages in order to bypass the impedance of the core (cf., Fig. 19(b)). For this purpose a copper foil with a thickness in the range of $20 \mu \mathrm{m}$ can be used or the ferrite material can be coated with copper by a galvanic process. As can be seen in Fig. 20 (measurement device Agilent 4294A) the utilization of the shield avoids the conduction losses in the ferrite material, the quality factor at high frequencies increases to the value corresponding to the $\mathrm{HF}$ winding resistance (e.g., at $f=1 \mathrm{MHz}$ from $Q=505$ to $Q=610$ ). It can also be noticed that the apparent increase of inductance value due to the occurring resonance is shifted slightly to a lower frequency in case of the shielded inductor because of the increase in winding capacitance of roughly $2 \mathrm{pF}$.

\section{APPENDIX D}

\section{Electrical Measurement of Surface Loss}

Several electrical core loss measurement techniques have been presented in literature and a comprehensive overview is given in [42]. In the conventional two-winding wattmeter method, the core loss is calculated with the measured primary current and the induced voltage across a second auxiliary winding used to exclude ohmic losses of the excitation winding from the power measurement. The measurement error derived in [41] for a given phase-shift between voltage and current, $\varphi_{2}$, and a phase discrepancy introduced by the instrumentation, $\Delta \varphi$, is given by

$$
\Delta=\tan \left(\varphi_{2}\right) \cdot \Delta \varphi \approx Q \cdot \Delta \varphi
$$

Thus, in order to minimize the potential measurement error caused by bandwidth limitations and/or uncompensated delays of the employed probes and to enable the characterization of high-Q, low-loss core materials at high frequencies, the sensitivity to phase errors is reduced by compensating the reactive impedance of the device under test (DUT) with a series connected capacitor or an air core inductor with opposing winding directions [41], [43]. A disadvantage of this technique is that add. power loss from the resonance capacitor (or air core inductor) is included in the power measurement and must be subtracted from the total loss based on a loss model of the component. The basic circuit schematic of the two-winding method with resonance capacitor is shown in Fig. 21(a) and typical waveforms for sinusoidal excitation are depicted in Fig. 21(b), showing that the aux. voltage $v_{\text {aux }}=v_{\mathrm{L}}+v_{\mathrm{C}}$ is in phase with the excitation current $i_{\mathrm{p}}$. According to literature, the resistance modeling the core losses is typ. drawn in series with the magnetizing inductance rather than in parallel since this facilitates a straightforward calculation of the losses for given primary current. Fig. 21(c) shows the test circuit employed in this study with additional sense winding with $N_{2}$ turns and a varistor at the input to protect the HF voltage source (Iwatsu IE$1125 \mathrm{~B}$ ) from over voltages. The sense winding is not used for the power instrumentation but to ensure the correct flux density amplitude in the sample as mentioned previously. For the twowinding wattmeter method to correctly exclude the winding losses, the excitation and auxiliary winding must feature close to ideal coupling. Now, the total losses measured with the test circuit are the sum of core losses in the E-type test circuit, $P_{\text {test }}$, the losses of the inserted multi-gap sample, $P_{\text {sample }}$, and the additional loss in the resonance capacitor, $P_{\text {cap }}$, 


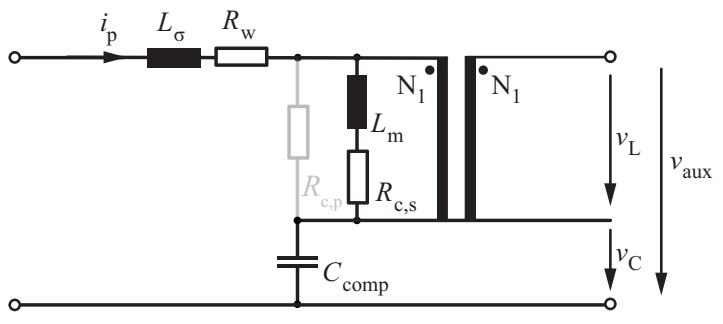

(a)

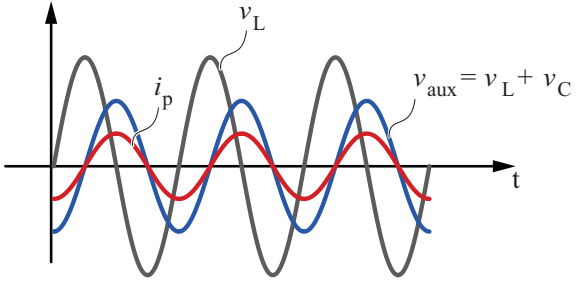

(b)

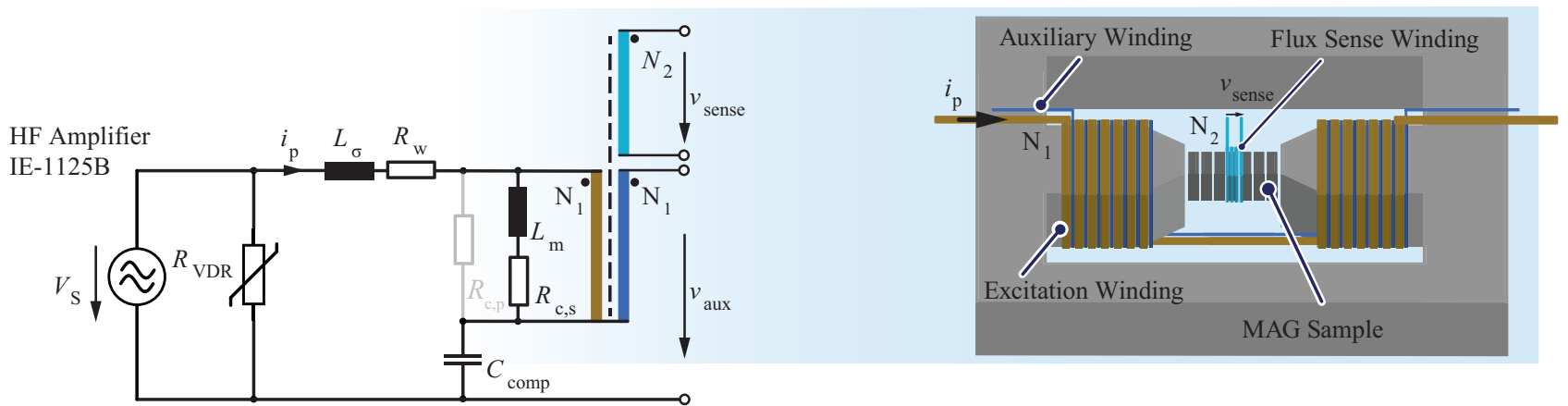

(c)

Fig. 21. (a) Circuit schematic of the two-winding wattmeter method with resonance capacitor and (b) typical waveforms for sinusoidal excitation showing that the aux. voltage $v_{\text {aux }}=v_{\mathrm{L}}+v_{\mathrm{C}}$ is in phase with the excitation current $i_{\mathrm{p}}$. (c) Electrical core loss test circuit used in this study with additional sense winding with $N_{2}$ turns and a varistor at the input to protect the HF voltage source; for the two-winding wattmeter method to correctly exclude the winding loss, the excitation and auxiliary winding must feature close to ideal coupling. The sense winding in this work is not used for the power instrumentation but to ensure the correct flux density amplitude in the sample.

$$
\begin{aligned}
P_{\text {tot }} & =\frac{1}{T_{\mathrm{m}}} \int_{0}^{T_{\mathrm{m}}} v_{\text {aux }} i_{\mathrm{p}} \mathrm{d} t \\
& =P_{\text {sample }}+P_{\text {test }}+P_{\text {cap }} .
\end{aligned}
$$

As proposed at the beginning of Section III, the total losses in the multi-gap core are the sum of the conventional core losses associated with the bulk ferrite material of the individual plates, $P_{\text {sample,c, }}$, and the surface related loss component, $P_{\text {sample,s }}$. It follows that,

$$
P_{\text {tot }}=P_{\text {sample }, \mathrm{s}}+P_{\text {sample }, \mathrm{c}}+P_{\text {core }}+P_{\text {cap }} \text {. }
$$

In order to extract $P_{\text {sample,s }}$ from the total measured loss the following idea is proposed. For a specific operating point $\{\hat{B}, f\}$, two loss measurements using multi-gap samples with distinct number of gaps are carried out. The samples are constructed in such a way that the total ferrite core volume remains constant and the individual gaps are adjusted to achieve identical reluctance. An example of two samples with 5 and 15 plates is provided in Table IV. It follows that for an identical reluctance of the two considered multi-gap samples, the equivalent reluctance of the entire magnetic test setup (test circuit and sample) remains constant and consequently the necessary excitation current to impress a specific flux density level in the sample will be equal. As a result, the losses of the test circuit and the resonance capacitor should ideally remain constant for a given operating point regardless of how many gaps are present in the installed sample. Now, the difference of two loss
TABLE IV

Two Distinct Samples With Identical Core and Total Gap Length

\begin{tabular}{cccc}
\hline \hline \# Gaps & Plate Thickness & Gap Length & Sample Length \\
\hline 4 & $3 \mathrm{~mm}$ & $175 \mu \mathrm{m}$ & $15.7 \mathrm{~mm}$ \\
14 & $1 \mathrm{~mm}$ & $50 \mu \mathrm{m}$ & $15.7 \mathrm{~mm}$ \\
\hline \hline
\end{tabular}

measurements with samples featuring a total of $u$ and $v$ gaps, is given by

$$
\begin{aligned}
\Delta P^{(\mathrm{u}, \mathrm{v})}= & P_{\text {tot }}^{\mathrm{u}}-P_{\text {tot }}^{\mathrm{v}}, \\
\stackrel{(25)}{=} & \left(P_{\text {sample, } \mathrm{s}}^{\mathrm{u}}+P_{\text {sample, } \mathrm{c}}^{\mathrm{u}}+P_{\text {test }}^{\mathrm{u}}+P_{\text {cap }}^{\mathrm{u}}\right)- \\
& \left(P_{\text {sample, } \mathrm{s}}^{\mathrm{v}}+P_{\text {sample, } \mathrm{c}}^{\mathrm{v}}+P_{\text {test }}^{\mathrm{v}}+P_{\text {cap }}^{\mathrm{v}}\right) \\
= & P_{\text {sample, } \mathrm{s}}^{\mathrm{u}}-P_{\text {sample, } \mathrm{s}}^{\mathrm{v}},
\end{aligned}
$$

wherein $P_{\text {sample, } \mathrm{c}}^{\mathrm{u}}=P_{\text {sample, } \mathrm{c}}^{\mathrm{v}}$ because the samples are designed to feature identical bulk volume (neglecting minus cule surface layer volume difference between samples), and $P_{\text {core }}^{\mathrm{u}}=P_{\text {core }}^{\mathrm{v}}$, $P_{\text {cap }}^{\mathrm{u}}=P_{\text {cap }}^{\mathrm{v}}$ because of the identical reluctance and excitation current as mentioned previously. The surface loss in a sample with $u$ gaps can furthermore be expressed as

$$
P_{\text {sample, } \mathrm{s}}^{\mathrm{u}}=\tilde{\kappa}_{\mathrm{s}}\left(2(u+1) \cdot A_{\mathrm{p}}\right),
$$

where $\tilde{\kappa}_{\mathrm{s}}$ is the surface loss density of plates with cross section 
$A_{\mathrm{p}}$. Inserting (29) in (26) and rearranging yields

$$
\tilde{\kappa}_{\mathrm{s}}=\frac{\Delta P^{(\mathrm{u}, v)}}{2(u-v) \cdot A_{\mathrm{p}}} .
$$

Thus by subtracting two measurements with identical operating point but using samples with different number of gaps the surface loss density can be extracted.

Unfortunately, the perfect cancellation of the test circuit power loss cannot be achieved in reality. Although it is quite challenging, it is possible to tune the gap length such that the impedance analyzer reads similar inductance values of the test circuit with either of the two samples installed. However, achieving a similar overall inductance value does not imply identical leakage flux conditions in the test circuit. Depending on the installed sample (cf., Table IV), a difference of up to $15 \%-20 \%$ of required magnetization current for a specific flux density amplitude was observed during first preliminary measurements.

Now, from (29) and (30) it can be seen that the measurement error of the surface loss density is directly determined by the error in computing $\Delta P$. The error in $\Delta P$ is not only affected by the error in the power instrumentation but also takes into account the systematic error arising from different leakage flux conditions in the test circuit and uncertainty in the ESR of the resonance capacitor which can be mitigated by using a large number of turns in the primary winding in order to keep current $i_{\mathrm{p}}$ low. Still, a rel. error of $15 \%-20 \%$ in the $P_{\mathrm{tot}}$ loss measurement is a reasonable estimate. Furthermore, for a sample with 15 plates and a mid-range operating point $\{125 \mathrm{mT}, 400 \mathrm{kHz}\}$ the actual surface loss amounts to just $20 \%-30 \%$ of the total measured power, $P_{\text {tot }}$. It follows then that the relative measurement error of $\widetilde{\kappa}_{\mathrm{s}}$ is likely in the range

$$
\frac{\sigma \widetilde{\kappa}_{\mathrm{s}}}{P_{\text {sample,s }}}=\frac{(10 \%-15 \%) \cdot P_{\text {tot }}}{(20 \%-30 \%) \cdot P_{\text {tot }}} \approx 33 \%-75 \%,
$$

which is too pronounced to obtain robust experimental results for a comparison of different ferrite materials and to examine the impact of different machining/manufacturing techniques and post-machining treatments on the surface loss. Since a direct measurement of the sample loss reduces the relative measurement error significantly, the thermometric measurement technique based on accurate temperature rise monitoring as discussed in Section III of this paper was developed.

\section{ACKNOWLEDGMENT}

The authors would like to thank F. Zajc for his suggestions and expertise regarding core loss measurements and the construction of MnZn ferrite samples (DMR 51, N87, PC200) analyzed in a preceding study. The authors grateful thanks are also extended to E. Hatipoglu for carrying out first electrical surface loss measurements (Appendix D). The microstructure analysis of the ferrite surface was carried out at ScopeM ETH Zurich and at EMPA Duebendorf. The help of G. Grossmann
(EMPA) in the FIB preparation and subsequent SEM anaylsis is very much appreciated by the authors. A big thank you furthermore goes to O. Perez from Ferroxcube for providing machined and directly sintered plates of the MnZn Ferrite material $3 \mathrm{~F} 4$ investigated in this paper.

\section{REFERENCES}

[1] J. Hu and C. R. Sullivan, "The Quasi-distributed gap technique for planar inductors: Design guidelines," in Proc. IEEE Ind. Appl. Conf., Oct. 1997.

[2] J. Hu and C. R. Sullivan, "AC resistance of planar power inductors and the quasidistributed gap technique," IEEE Trans. Power Electron., vol. 16 , no. 4, pp. 558-567, 2001 .

[3] F. Zajc, "Multi gap inductor core, multi gap inductor, transformer and corresponding manufacturing method and winding," European Union Patent Application EP 2528069 A1, Nov., 2012.

[4] Z. Franc, "Flat band winding for an inductor core," U.S. Patent US2 012 299 681(A1), Nov., 2012.

[5] Z. Franc, "Winding arrangement for inductive components and method for manufacturing a winding arrangement for inductive components," U.S. Patent US2 015325 361(A1), Nov., 2015.

[6] D. Bortis, D. Neumayr, and J. W. Kolar, “ $\eta p$-pareto optimizationand comparative evaluation of inverter concepts considered for thegoogle little box challenge," in Proc. 17th IEEE Workshop Control Modeling Power Electron. (COMPEL), June 2016.

[7] GOOGLE, "Detailed inverter specifications, testing procedure, andtechnical approach and testing application requirements for the littlebox challenge," GOOGLE, Tech. Rep., 2015. [Online]. Available: https://www.littleboxchallenge.com

[8] C. R. Sullivan, H. Bouayad, and Y. Song, "Inductor design for lowloss with dual foil windings and quasi-distributed gap," in Proc. IEEE Energy Convers. Congr. Expo. (ECCE US), 2013.

[9] C. R. Sullivan, "Layered foil as an alternative to litz wire: multiplemethods for equal current sharing among layers," in Proc. 15th IEEE Workshop Control Modeling Power Electron. (COMPEL), 2014.

[10] E. Stern and D. Temme, "Magnetostriation effects in remanence phase shifters," IEEE Trans. Microwave Theory Techn., vol. 13, no. 6, pp. 873-874, Nov. 1965.

[11] J. E. Knowles, "The effect of surface grinding upon the permeabilityof manganese - Zinc ferrites," J. Physics D: Appl. Physics, vol. 3, no. 9, pp. $1346,1970$.

[12] J. Knowles, "The origin of the increase in magnetic loss induced by machining ferrites," IEEE Trans. Magn., vol. 11, no. 1, pp. 44-50, Jan. 1975.

[13] E. Snelling, "The effects of stress on some properties of MnZn ferrite," IEEE Trans. Magn., vol. 10, no. 3, pp. 616-618, Sep. 1974.

[14] E. Klokholm and H. L. Wolfe, Surface Damage in Manganese Zincand Nickel Zinc Ferrites. Dordrecht: Springer Netherlands, 1984, pp.665-681.

[15] S. Chandrasekar and B. Bhushan, "Comparison of grinding and lapping of ferrites and metals," J. Eng. Ind., vol. 109, no. 2, pp. 76-82, 1987.

[16] S. Chandrasekar and B. Bhushan, "Control of surface finishing residual stresses in magnetic recording head materials," J. Tribol., vol. 110, no. 1, pp. 87-92, 1988 .

[17] B. Bhushan, Tribology and mechanics of magnetic storage devices, 2nd ed. Springer-Verlag, 1990.

[18] H. Naumoski, B. Riedm"uller, A. Minkow, and U. Herr, "Investigation of the influence of different cutting procedures on the global and local magnetic properties of non-oriented electrical steel," J. Magnetism \& Magn. Materials, vol. 392, pp. 126-133, 2015.

[19] M. Hofmann, H. Naumoski, U. Herr, and H. G. Herzog, "Magnetic properties of electrical steel sheets in respect of cutting: micromagnetic analysis and macromagnetic modeling," IEEE Trans. Magn., vol. 52, no. 2, pp. 1-14, 2016.

[20] M. Bali, H. De Gersem, and A. Muetze, "Determination of original nondegraded and fully degraded magnetic characteristics of material subjected to laser cutting," IEEE Trans. Ind. Appl., vol. 53, no. 5, pp. 4242-4251, 2017. 
[21] V. Loyau, M. Lo Bue, and F. Mazaleyrat, "Measurement of magnetic losses by thermal method applied to power ferrites at high level of induction and frequency," the Review of Scientific Instruments, vol. 80, no. 2, 2009 .

[22] T. Komma and H. Gueldner, "A method of determining core losses caused by a DC flux-density bias," in Proc. Int. Exhibition Conf. Power Electron, Intell. Motion, Renewable Energy (PCIM Europe), Nuremberg, Germany, 2002.

[23] V. Loyau, M. Lobue, and F. Mazaleyrat, "Comparison of losses measurement in a ferrite with two calorimetric methods," IEEE Trans. Magn., vol. 46, no. 2, pp. 529-531, 2010.

[24] L. Ferraris, F. Franchini, and E. Pokovi, "A thermographic method for the evaluation of the iron losses distribution in electromagnetic devices," in Proc. 42nd Annu. Conf. IEEE Ind. Electron. Soc. (IECON), Oct. 2016.

[25] H. Shimoji, B. E. Borkowski, T. Todaka, and M. Enokizono, "Measurement of core-loss distribution using thermography," IEEE Trans. Magn., vol. 47, no. 10, pp. 4372-4375, Oct. 2011.

[26] L. Ferraris, E. Poskovic, and F. Franchini, "Defects detection in laminated and soft magnetic composites devices with a novel thermographic method," in Proc. 11th IEEE Int. Symp. Diagnostics Elect. Machines Power Electron. Drives(SDEMPED), Aug. 2017.

[27] H. Shimoji, B. E. Borkowski, T. Todaka, and M. Enokizono, "Visualizing iron loss distribution in permanent magnet motors," in Proc. 15th IEEE Int. Conf. Elect. Mach. Syst. (ICEMS), Oct. 2012.

[28] FLIR, "FLIR A655sc Infrared Camera Datasheet," Brochure, 2014. [Online]. Available: http://www.flirmedia.com/MMC/THG/Brochures/ RNDn 011/RNDn 011n US.pdf

[29] Infrared Camera Accuracy and Uncertainty in Plain Language, Tech Note, 2016. [Online]. Available: https://www.flir.com/discover/rd-science/ infrared-camera-accuracy-and-uncertainty-in-plain-language/

[30] A. A. Clifford, Multivariate Error Analysis: A Handbook of Error Propagation and Calculation in Many-Parameter Systems. Wiley, 1973.

[31] Ferroxcube, "Ferroxcube Software Design Tool (SFDT 2010)," [Online]. Available: https: //www.ferroxcube.com/en-global/design tool/index, 2010, last accessed 2018-04-23.

[32] C. A. Baguley, U. K. Madawala, and B. Carsten, "Unusual effects measured under DC bias conditions on MnZn ferrite material," IEEE Trans. Magn., vol. 45, no. 9, pp. 3215-3222, 2009.

[33] C. A. Baguley, U. K. Madawala, and B. Carsten, "The impact of vibration due to magnetostriction on the corelosses of ferrite toroidals under DC bias," IEEE Trans. Magn., vol. 47, no. 8, pp. 2022-2028, 2011.

[34] C. A. Baguley, U. K. Madawala, B. Carsten, and M. Nymand, "The impact of magnetomechanical effects on ferrite B-H loop shapes," IEEE Trans. Magn., vol. 48, no. 8, pp. 2284-2292, 2012.

[35] R. S. Yang, A. J. Hanson, D. J. Perreault, and C. R. Sullivan, "A low-loss inductor structure and design guidelines for high-frequency applications," in Proc. IEEE Appl. Power Electron. Conf. Expo. (APEC), Mar. 2018.

[36] Duralco, "Duralco/Durapot Epoxide," [Online]. Available: http://www. polytec-pt.com/de/produkte/hochtemperaturklebstoffe-und-keramischematerialien/hochtemperatur-epoxide/,2010, last accessed 2018-03-26.

[37] H. Meuche, A. Nguyen, D. Lange, and M. Esguerra, "Influence of a mechanical stress on ferrite materials for broadband applications," in Proc. 9th Int. Conf. Ferrites (ICF-9), 2004

[38] V. Tsakaloudi, D. Holz, and V. Zaspalis, "The effect of externally applied uniaxial compressive stress on the magnetic properties of power $\mathrm{MnZn}$ ferrites," J. Material Sci., vol. 48, no. 10, pp. 3825-3833, May 2013.

[39] M. Baumann, S. Sotier, and J. Weidinger, "A model of initial permeability of soft ferrite in dependence of biaxial stress," J. Japan Soc. Powder \& Powder Metallurgy, vol. 61, no. S1, pp.70-74, May 2014

[40] T. Thomas, S. Hoffmann, K.-F. Becker, H. Walter, V. Bader, T. Braun,E. Hoene, and M. Schneider-Ramelow, "Ferrite embedding for power SiPs A packaging view," in Proc. 10th IEEE Conf. Integrated Power Electron. Syst. (CIPS), 2018.

[41] M. Mu, Q. Li, D. J. Gilham, F. C. Lee, and K. D. T. Ngo, "New core loss measurement method for high-frequency magnetic materials," IEEE Trans. Power Electron., vol. 29, no. 8, pp. 4374 4381, Aug. 2014.

[42] C. R. Sullivan, "Survey of Core Loss Test Methods," 2017. [Online]. Available: http://sites.dartmouth.edu/power-magnetics/files/2017/03/ Survey-of-Core-Loss-Test-Methods-Sullivan.pdf

[43] D. Hou, M. Mu, F. C. Lee, and Q. Li, "New high-frequency core loss measurement method with partial cancellation concept," IEEE Trans. Power Electron., vol. 32, no. 4, pp. 2987-2994, Apr. 2017.

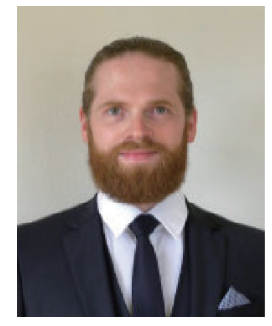

Dominik Neumayr started his academic education at the University of Applied Sciences (FH) for Automation Engineering in Wels, Austria and received the Dipl.-Ing. (FH) degree in 2008.

He was with the Center for Advanced Power Systems (CAPS) in Tallahassee, FL, USA, working on Power/Controller Hardware-in-the-Loop Simulations and Control Systems Design for AC/DC/AC PEBB based converter systems from ABB. He continued his academic education at the Swiss Federal Institute of Technology in Zurich (ETH Zurich) with a focus on power electronics and control engineering and received the M.Sc. degrees in Electrical Engineering and information technology in 2015. Since spring 2015 he is a Ph.D. student at the Power Electronic Systems (PES) Laboratory, ETH Zurich. His current research focuses on the design and control of high power density converter systems.

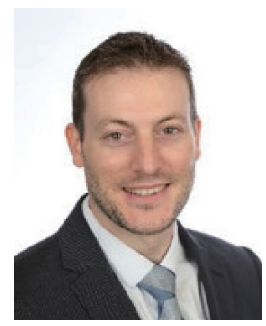

Dominik Bortis received the M.Sc. degree in electrical engineering and the Ph.D. degree from the Swiss Federal Institute of Technology (ETH) Zurich, Switzerland, in 2005 and 2008, respectively. In May 2005, he joined the Power Electronic Systems Laboratory (PES), ETH Zurich, as a Ph.D. student. From 2008 to 2011, he has been a Post-doctoral Fellow and from 2011 to 2016 a Research Associate with PES, co-supervising Ph.D. students and leading industry research projects. Since January 2016 Dr. Bortis is heading the newly established research group Advanced Mechatronic Systems at PES.

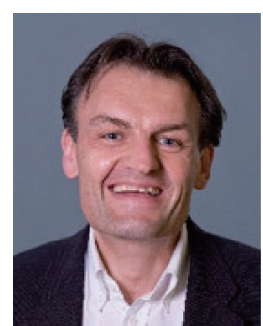

Johann W. Kolar received his M.Sc. and Ph.D degree (summa cum laude / promotio sub auspiciis praesidentis rei publicae) from the University of Technology Vienna, Austria, in 1997 and 1999, respectively. Since 1984, he has been working as an independent researcher and international consultant in close collaboration with the University of Technology Vienna, in the fields of power electronics, industrial electronics and high performance drives. $\mathrm{He}$ has proposed numerous novel PWM converter topologies, and modulation and control concepts and has supervised over $70 \mathrm{Ph}$.D. students. He has published over 650 scientific papers in international journals and conference proceedings, 3 book chapters, and has filed more than 160 patents. The focus of his current research is on ultra-compact and ultra-efficient $\mathrm{SiC}$ and $\mathrm{GaN}$ converter systems, wireless power transfer, solid-state transformers, power supplies on chip, and ultra-high speed and bearingless motors. Dr. Kolar has received 27 IEEE Transactions and Conference Prize Paper Awards, the 2014 IEEE Middlebrook Award, and the ETH Zurich Golden Owl Award for excellence in teaching. He initiated and/or is the founder of four ETH Spin-off companies. He is a member of the steering committees of several leading international conferences in the field and has served from 2001 through 2013 as an associate editor of the IEEE Transactions on Power Electronics. Since 2002 he also is an associate editor of the Journal of Power Electronics of the Korean Institute of Power Electronics and a member of the Editorial Advisory Board of the IEEJ Transactions on Electrical and Electronic Engineering. 


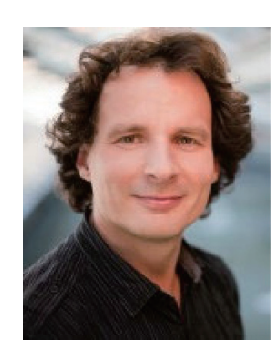

Stefan Hoffmann successfully completed his M.Sc. at the Technical University Berlin, Germany in 2010. At the beginning of 2011 he was assigned as a research assistant to the Power Electronics working group at Fraunhofer Institute for Reliability and Microintegration, Berlin, Germany. His current research interests include investigations on magnetic materials, design of power electronic systems and EMC.

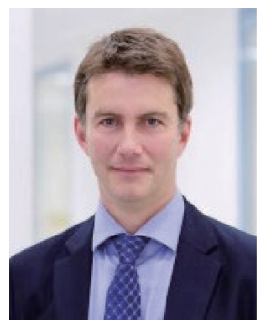

Eckart Hoene received his Diploma degree in Electrical Engineering from the Technical University Berlin in 1997. He then joined Fraunhofer Institute for Reliability and Microintegration, Berlin, Germany as scientific assistant and worked in parallel towards his Ph.D., which he received in 2001 from the TU Berlin. He continued at Fraunhofer as Postdoc, group leader and business development manager. In 2014 he became adjunct professor at Aalborg University, in addition to the courses he conducts for the European Center for Power Electronics (ECPE) and his Fraunhofer affiliation. His technical interests are in high switching frequency power electronics, semiconductor packaging and EMC. 